\title{
Cerebral White Matter Myelination and Relations to Age, Gender, and Cognition: A Selective Review
}

\author{
Irina S. Buyanova ${ }^{1,2}$ and Marie Arsalidou ${ }^{1,3,4 *}$ \\ ${ }^{1}$ Neuropsy Lab, HSE University, Moscow, Russia, ${ }^{2}$ Center for Language and Brain, HSE University, Moscow, Russia, \\ ${ }^{3}$ Cognitive Centre, Sirius University of Science and Technology, Sochi, Russia, ${ }^{4}$ Department of Psychology, York University, \\ Toronto, ON, Canada
}

\section{OPEN ACCESS}

Edited by:

Torsten Wüstenberg, Heidelberg University, Germany

Reviewed by: Michela Ferrucci, University of Pisa, Italy Robert Turner

Max Planck Institute for Human Cognitive and Brain Sciences, Germany

*Correspondence: Marie Arsalidou marie.arsalidou@gmail.com

Specialty section:

This article was submitted to Cognitive Neuroscience, a section of the journal Frontiers in Human Neuroscience

Received: 31 January 2021 Accepted: 02 June 2021 Published: 06 July 2021

Citation:

Buyanova IS and Arsalidou M (2021) Cerebral White Matter Myelination and Relations to Age, Gender, and Cognition: A Selective

Review.

Front. Hum. Neurosci. 15:662031. doi: 10.3389/fnhum.2021.662031
White matter makes up about fifty percent of the human brain. Maturation of white matter accompanies biological development and undergoes the most dramatic changes during childhood and adolescence. Despite the advances in neuroimaging techniques, controversy concerning spatial, and temporal patterns of myelination, as well as the degree to which the microstructural characteristics of white matter can vary in a healthy brain as a function of age, gender and cognitive abilities still exists. In a selective review we describe methods of assessing myelination and evaluate effects of age and gender in nine major fiber tracts, highlighting their role in higher-order cognitive functions. Our findings suggests that myelination indices vary by age, fiber tract, and hemisphere. Effects of gender were also identified, although some attribute differences to methodological factors or social and learning opportunities. Findings point to further directions of research that will improve our understanding of the complex myelinationbehavior relation across development that may have implications for educational and clinical practice.

Keywords: white matter, myelination, fiber tracts, brain development, cognitive abilities, magnetic resonance imaging, diffusion tensor imaging

\section{INTRODUCTION}

Humans can move, think and feel in part because their brain communicates signals through neurons. Neurons align themselves in brain layers such that gray matter consists of cell bodies and dendrites, and white matter (WM) consists of neuronal fibers with varying degree of myelination that allows transfer of signals across distributed brain areas. WM accounts for more than half of the total brain volume (Fields, 2010) and is involved in cognitive (Desmond, 2002; Ohlhauser et al., 2018), affective (Zhang et al., 2018), sensory (Pryweller et al., 2014; Chang et al., 2016), and motor functions (Sampaio-Baptista et al., 2013; Fleischman et al., 2015; Hollund et al., 2017). Myelination of cerebral WM changes with individual differences such as age (i.e., maturation) and learning (i.e., experience; Fields, 2010; Filley and Fields, 2016) and may be gender-specific (Passe et al., 1997; den Braber et al., 2013; Bourisly et al., 2017).

Myelination of neuronal axons in the gray matter (GM) of the brain is represented by radial and horizontal axons. Radial axonal fibers extend to the cytoarchitectonic Layer II and can pass long distances (up to $2 \mathrm{~mm}$ ) connecting pyramidal neurons from the deeper layers with the cortical surface (Vogt and Vogt, 1919). Horizontal or tangential fibers are short-distance cortical axons, which originate mainly from inhibitory interneurons (Micheva et al., 2016) and lie in the 
two bands of Baillarger (1840) in cortical layers IV and V, in Exner's stripe within the molecular Layer I (Stepanyants et al., 2009; Leuze et al., 2014), and late-myelinated $U$-fibers located within the superficial WM outside the GM (Leuze et al., 2014). Radial fibers can contain unmyelinated axons, which form temporary synaptic connections to local dendrites. Rate of myelination in short association fibers is region-specific (Flechsig, 1920), and is concurrent with the developmental trajectories of cognitive and motor skills (Turner, 2019).

The speed and timing of myelination of neuronal axons within association, projection, and commissural fiber tracts that comprise cerebral WM reflect their position in the functional hierarchy (Yakovlev and Lecours, 1967). For example, myelination of the motor root fibers is faster and shorter than that of sensory roots. In an adult brain, there are three myeloarchitectonic regions, which myelinate with different cycles as distinct tectogenetic units. A median periventricular zone comprises the median thalamus and the hypothalamus, the hippocampus, and the hippocampal rudiment and septal area. A paramedian (limbic) zone includes the paramedian thalamus, subthalamic region, the internal capsule, and the pallidum and striatum with the amygdala and claustrum. A supralimbic zone, including the bulk of the WM of the frontal, parietal, and temporal lobes and respective opercula.

Quantitative evaluation of WM volume and microstructural properties of myelinated fiber bundles is critical for understanding developmental trajectories in health and disease. Progressive myelination of WM pathways throughout infancy, childhood, and adolescence and into adulthood is concurrent with pronounced changes in cognitive abilities due to more rapid neural communication and integration of the information across functionally related brain regions (Giedd et al., 1999; Paus, 2010; Lebel and Beaulieu, 2011; Arshad et al., 2016; Morris et al., 2020).

White matter pathways in the brain are largely represented by association, commissural and projection fibers (Mandonnet et al., 2018). Association fibers connect close and distant brain areas within the same hemisphere and include the superior longitudinal fasciculus (SLF), arcuate fasciculus (AF), cingulum, inferior longitudinal fasciculus (ILF), inferior fronto-occipital fasciculus (IFOF), uncinate fasciculus (UF), middle longitudinal fasciculus (MdLF), and frontal aslant tract (FAT) (Catani and Thiebaut de Schotten, 2012; Gupta, 2017).

Commissural pathways connect the two hemispheres and are represented by the corpus callosum (CC), the anterior and posterior commissures, the hippocampal commissure, the habenular commissure, and the hypothalamic and cerebellar commissures (Catani and Thiebaut de Schotten, 2012; Gupta, 2017). Commissural pathways play a critical role in the integration and transfer of the information between the motor, perceptual, and cognitive modalities. Projection fibers pass through the internal capsule, corona radiata, cerebral peduncles, and brainstem to connect the cerebral cortex to other subcortical structures, such as spinal cord, deep cerebral nuclei, and brainstem nuclei, forming bidirectional (i.e., ascending and descending) connections. Output from the hippocampus to the cortex is formed by the fornix, which begins in the hippocampus and passes longitudinally toward the diencephalon and basal forebrain, arching over the thalamus (Catani and Thiebaut de Schotten, 2012; Gupta, 2017). Myelination cycles of the association pathways are protracted compared to commissural and projection fibers, as they connect cortical regions, providing functional coherence between brain areas.

Many studies focused on WM development (Lebel and Beaulieu, 2011; Sexton et al., 2014; Lebel and Deoni, 2018; Tamnes et al., 2018) and effects of WM lesions and cognitive impairments (Desmond, 2002; Valdés Hernández et al., 2013; Aso et al., 2017). Fewer studies investigate typical maturation of WM tracts in relation to cognitive functions. Literature associated with cerebral WM covers various pockets of knowledge such as methods of assessment, different conceptions of anatomical characteristics of fiber tracts, developmental effects, gender effects, and their association to mental function. Critically, a synthesis of the literature is lacking and aggregation of topics can help generate hypotheses for future research and create links between practice and research. Due to space limitations this review focuses on the most popular methods for assessing myelination, identifies anatomical considerations of nine fiber tracts, and presents current knowledge on developmental trajectories in relation to individual differences associated with age, gender, and cognition. Specifically, we cover eight major association tracts, SLF, AF, cingulum, ILF, IFOF, UF, MdLF, FAT; and one major commissural fiber system, the $\mathrm{CC}$, which plays a critical role in the interhemispheric integration and transfer of the information. Although different methods exist that directly or indirectly assess microstructural properties of the WM, the most popular at this time is diffusion tensor imaging (DTI), thus, the review of individual differences related to age, gender and cognition focuses mainly on the DTI metrics, emphasizing its advantages, disadvantages, and research gaps for future studies.

\section{METHODS FOR ASSESSING MYELINATION}

The classic methods used for myelination assessment and investigation of WM was postmortem histological staining with hematoxylin/eosin (Flechsig, 1901; Yakovlev and Lecours, 1967), the Luxol fast blue technique (Bodhireddy et al., 1994), and subsequent immunohistochemical staining for myelin basic protein and myelin-associated glycoprotein (Itoyama et al., 1980). Critically, these methods cannot be used for in vivo studies. The development of non-invasive magnetic resonance imaging (MRI) techniques made it possible to indirectly assess myelination and other microstructural indices in the living brain. These methods include DTI, Myelin Water Imaging (MWI), and Magnetization Transfer Imaging (MTI), g-ratio imaging, and myelin mapping.

\section{Diffusion Tensor Imaging}

Diffusion tensor imaging is one of the most popular noninvasive indirect imaging methods used to assess microstructure and orientation of myelinated WM fibers (Basser et al., 1994; Basser and Jones, 2002; Alexander et al., 2007). Diffusion MRI (dMRI) allows for investigation of the microstructure of the tissues by measuring diffusion of water molecules 
within the tissue considering their interaction with biological membranes and macromolecules and has been widely used to study individual differences in WM microstructure related to neurological and psychiatric conditions, as well as in healthy brains (Forkel et al., 2020).

Water diffusion can be characterized by the diffusion coefficient, $\boldsymbol{D}, \quad\left(\mathrm{mm}^{2} / \mathrm{s}\right)$, which links diffusive flux and concentration gradient (Doran et al., 1996; Johansen-Berg and Behrens, 2009). Diffusion of water molecules in a solution is influenced by intermolecular interactions, temperature, molecular weight, and various active processes within the tissue (Beaulieu, 2002). Isotropic diffusion is described by a single apparent diffusion coefficient, which characterizes the interaction between the diffusing molecules and cellular structures (Basser and Jones, 2002; Beaulieu, 2002). In the brain's WM, especially in highly myelinated regions, water diffusion is anisotropic (i.e., it is restricted by axonal membranes; Beaulieu, 2002) and can be described by a diffusion tensor, which replaces the scalar diffusion coefficient (Basser and Jones, 2002). The main direction of anisotropic diffusion within WM reflects fiber orientation, and, therefore, structural characteristics of a given brain region can be quantitatively determined by measuring water diffusion.

The displacement of the molecules is modeled using threedimensional Gaussian distribution (O'Donnell and Westin, 2011). The diffusion tensor model is fitted using multi-linear regression; to estimate diffusion tensor, diffusion gradients must be applied at least along six noncollinear, noncoplanar directions (Basser et al., 1994). The diffusion tensor determines three orthogonal eigenvectors and associated eigenvalues $\left(\lambda_{1}, \lambda_{2}\right.$, and $\left.\lambda_{3}\right)$, which describe the diffusivity in the direction of each eigenvector. The direction of the main eigenvector corresponds to the direction of the fastest diffusion and determines the axis of a given fiber tract in the regions where the WM tracts do not branch and cross (Mori and Zhang, 2006; O’Donnell and Westin, 2011).

Diffusion tensor may be geometrically represented as an ellipsoid whose axes are of the length $\sqrt{2} \tau \lambda_{i}$ and are aligned with the eigenvectors (Basser et al., 1994). The eigenvalues of the diffusion tensor can be used to estimate widely used diffusion parameters, including (1) the mean diffusivity (MD), which reflects the total diffusion in a voxel, (2) longitudinal or axial diffusivity (AD), which is equal to the eigenvalue corresponding to the longest axis $\left(\lambda_{\|} \lambda_{1}\right.$, assuming $\left.\lambda_{1} \geq \lambda_{2} \geq \lambda_{3} \geq 0\right)$, (3) transverse or radial diffusivity (RD), which reflects the diffusion in the transverse plane and may indicate changes in axonal diameter, and (4) fractional anisotropy (FA), which characterizes the eccentricity of the diffusion ellipsoid and provides quantitative measure of the shape of the diffusion ranging from 0 to 1 (Basser and Jones, 2002; O'Donnell and Westin, 2011). $\mathrm{MD}$ is an inverse measure of membrane density and spacing between axons, which reflects the directionally invariant overall diffusion rate (Beaulieu, 2014). Notably, MD is similar in white and gray matter (Basser and Pierpaoli, 1996; Beaulieu, 2014). FA reflects the difference in the shape of the tensor ellipsoid between a perfect sphere and an ellipsoid that describes anisotropic diffusion.
The diffusion of water molecules is measured using the pulsedgradient, spin echo pulse sequence with a single-shot echoplanar imaging (Stejskal and Tanner, 1965; Westin et al., 2002; Alexander et al., 2007). Fiber orientation may be visualized using color-coding in which blue color corresponds to superior to inferior direction, red color reflects left to right orientation, and green denotes anterior to posterior direction (Pajevic and Pierpaoli, 1999). FA values are usually reflected by the brightness of the color (O'Donnell and Westin, 2011). Fiber trajectories are assessed using dMRI tractography (Basser et al., 2000), given that the orientation of the major eigenvector of the diffusion tensor is parallel to the fiber tracts (Figure 1). dMRI tractography starts at a "seed" point, that is a specified location at which the direction of the principal eigenvector is measured followed by moving a small fixed distance ( $\leq 1 \mathrm{~mm}$ ) in that direction (tract integration). Evaluation of the fiber orientation is repeated and re-evaluated for successive small steps until the tract is terminated (Alexander et al., 2007). dMRI tractography has a number of limitations, including the susceptibility of estimates of eigenvector directions to thermal noise, physiologic fluctuations, and image artifacts. In addition, this method is based on the assessment of the principal eigenvector, thus making it impossible to study the direction of the fiber tracts that branch or cross (Alexander et al., 2007; O'Donnell and Westin, 2011). Nonetheless, this technique has important advantages for imaging brain structures in vivo and is widely used in normative and clinical studies. Advances in scanning sequences and analysis approaches make DTI one of the most popular methods for assessing WM microstructure and myelination.

\section{Myelin Water Imaging}

Myelin Water Imaging is based on evaluation of myelin water fraction (MWF), which serves as a sensitive marker for myelin

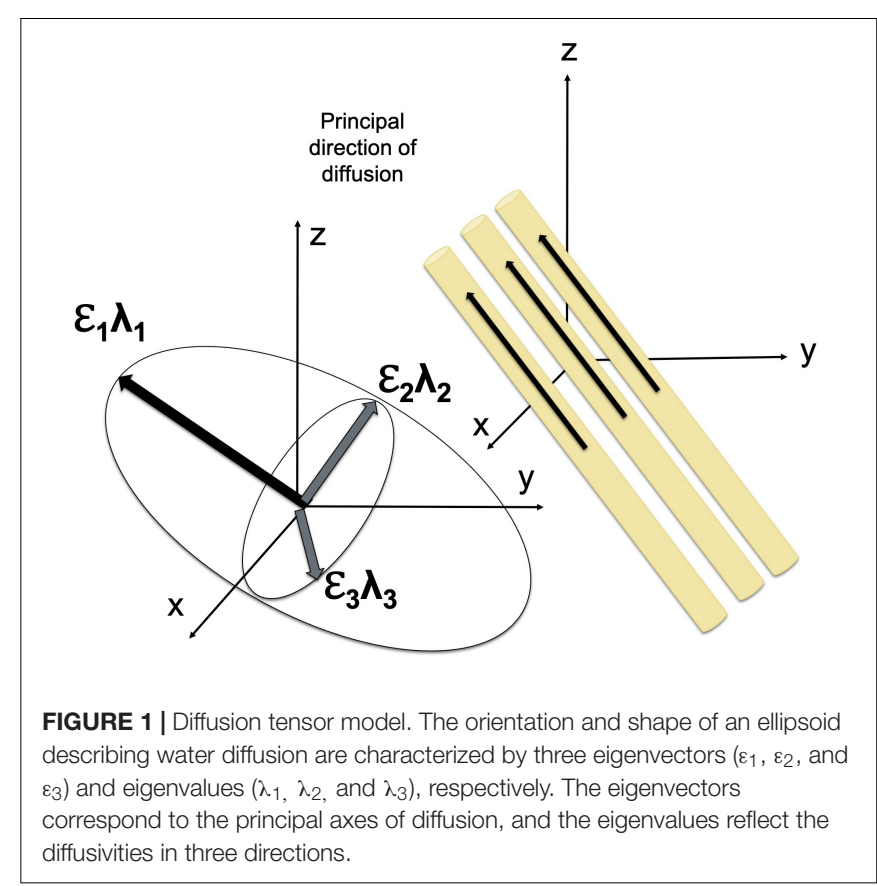


content. MWF corresponds to the fraction of water trapped by the myelin lipid bilayer (MacKay et al., 1994; Whittall et al., 1997; Deoni et al., 2012; MacKay and Laule, 2016) and allows for quantitative assessment of changes in fiber myelination (Deoni et al., 2011, 2012; Morris et al., 2020). MWI uses multi-exponential $\mathrm{T}_{2}$ relaxation data acquired with a multi-echo Gradient and Spin Echo sequence (MacKay and Laule, 2016; Faizy et al., 2020; Morris et al., 2020). The transverse relaxation time ( $T_{2}$, spin-spin relaxation time) determines the decay of transverse magnetization - the rate at which the spinning protons go out of phase with each other, i.e., lose phase coherence among the nuclei spinning perpendicularly to the main magnetic field B0. The transverse relaxation is related to the intrinsic field caused by the nearby protons. The individual nuclei within the tissue of interest precess in the transverse plane at slightly different rates so that their magnetic moments point to different directions and randomly interact with each other at the atomic or molecular level leading to the transverse decay of the MR signal and irreversible dephasing of the transverse magnetization. The exponential signal decay is therefore influenced by surrounding protons, and $T_{2}$ for water trapped by the myelin sheath is shorter than $\mathrm{T}_{2}$ for extracellular or intracellular water, and decay amplitude is proportional to water amount in each medium.

Myelin Water Imaging is obtained from $\mathrm{T}_{2}$ spectrum after the extraction of exponential components in a $\mathrm{T}_{2}$ decay curve and is computed in each voxel as the ratio between the $\mathrm{T}_{2}$ distribution area corresponding to myelin water $\left(\mathrm{T}_{2}<40-50 \mathrm{~ms}\right)$ to the total $\mathrm{T}_{2}$ distribution area, and thus is independent from the magnetic field. Fitting $T_{2}$ decay with other $T_{2}$ components allows for the extraction of the signal derived from myelin water with short $\mathrm{T}_{2}$ (Whittall et al., 1997; MacKay and Laule, 2016; Morris et al., 2020). At 3 Telsa, signal from myelin water has the shortest $\mathrm{T}_{2}$ component $(<40-50 \mathrm{~ms}$; MacKay et al., 1994; Whittall et al., 1997; Deoni et al., 2012). This algorithm can also be applied to calculate $\mathrm{T}_{2}{ }^{*}$ and $\mathrm{T}_{1}$ spectra (Hwang et al., 2004; Labadie et al., 2014).

The multicomponent model of relaxation signals evaluates the relative contributions of and interaction between multiple components, including intra- and extra-cellular water, cerebrospinal fluid, and myelin-related components (i.e., the water molecules within the myelin lipid bilayer; Lancaster et al., 2003; Deoni et al., 2013). However, to fit this model, a large number of measurements are needed leading to long acquisition times. Further, sophisticated preprocessing algorithms and long post-processing times impede the implementation of this method in pediatric imaging. Although new acquisition algorithms and processing strategies have been proposed, they still need to be validated before being introduced to routine clinical practice (Kulikova, 2015).

In agreement with postmortem data documenting myelination progress with age (Kaes, 1907), MWF increases in a caudo-rostral direction, following a parabolic trajectory with age. Changes in DTI indices usually exhibit linear association with age, and in studies which report non-linear relations peak age of myelination often depends on inclusion of young participants in selected WM regions (Westlye et al., 2010; Lebel et al., 2012). Indeed, peak age of myelination assessed by DTI indices is observed between 24 and 39 years (Sexton et al., 2014; Arshad et al., 2016), which is earlier than reported in MWI studies (Kochunov et al., 2012; Lebel et al., 2012) and MWF studies (Flynn et al., 2003; Arshad et al., 2016). The effect of age on DTI metrics is often different from that on MWF measures, showing weak or no correlations, as DTI may reflect other WM properties and components; therefore, some suggest that individual differences in myelination cannot be accurately described by variation in DTI-derived indices (Billiet et al., 2015; Arshad et al., 2016).

As evidenced by developmental studies, changes in MWF usually show quadratic association with age, as has also been indicated in postmortem studies (Arshad et al., 2016). Although DTI does show sensitivity to age-related changes in the cerebral WM, it may not be specific to myelin content (Jones et al., 2013a; Faizy et al., 2020). DTI metrics are significantly influenced by fiber density and orientation, as well as spatial organization (i.e., packing and crossing) and number of axons, whereas MWF reflects more closely myelin content, a hypothesis that has been validated histologically (Morris et al., 2020). However, the number of MWI studies investigating age-related changes in WM microstructure is limited (Dean et al., 2015; Arshad et al., 2016; Lynn et al., 2019), and recently developed MWI methods have not been validated with large normative samples and clinical settings. The computation of the MWF is also very sensitive to the acquisition parameters and computational settings, which makes it hard to compare results across studies.

\section{Magnetization Transfer Imaging}

Magnetization transfer imaging is based on quantitative evaluation of magnetization exchange between spins via chemical exchange or cross-relaxation caused by spin diffusion (Edzes and Samulski, 1978; Fung, 1986). Spin diffusion is the quantum mechanical process by which spins can flip in a rigid soil medium and is associated with coupling between spinlattice relaxations of different nuclei, such as macromolecular protons and water protons, in the presence of motion (Derome, 1987). Due to ultra-short transverse relaxation time $T_{2}$ and low mobility of macromolecular protons associated with proteins and lipids, such as the galactocerebrosides within myelinated membranes (Kucharczyk et al., 1994; Engelbrecht et al., 1998), relaxation time of macromolecular spins cannot be directly visualized using MRI (Henkelman et al., 2001). Coupling between mobile water protons and protons associated with macromolecules results in exchange (i.e., magnetization transfer, MT), which allows for indirect imaging of these less mobile spins within the macromolecular proton fraction (Tozer et al., 2003).

The effect of cross relaxation in a heterogenous biological system is traditionally studied in the context of a two-pool model, which implies that a given biological system is comprised of two pools - the water protons (free pool), and protons associated with macromolecules (bound pool). These pools differ in their transverse relaxation times, with the transverse relaxation time of macromolecular protons and protons associated with biological membranes to be much smaller $(<1 \mathrm{~ms})$ than that of water $(>10 \mathrm{~ms})$. When two protons are close to each 
other, cross-relaxation between them may take place, resulting in simultaneous flips of spins via dipolar magnetic interaction. The spin-lattice relaxation in each pool is determined by a sum of two exponential decays characterized by two apparent relaxation rates.

The excitation of the proton spins bound to macromolecules by a radiofrequency pulse results in the transfer the energy to the nearby proton spins, which is referred to as the magnetization transfer effect (Henkelman et al., 2001). Traditionally, the MT effect has been assessed using an MT ratio (MTR), which measures a relative decrease in observed signal in the saturation experiment with an off-resonance radiofrequency pulse (Yarnykh, 2012) and allows for the evaluation of myelin content in the WM. Based on MT-weighted, $\mathrm{T}_{1}$-weighted, and proton-density weighted images, it is possible to build macromolecular proton fraction (MPF) maps, which describe the proportion of macromolecular protons contributing to MT effect as a result of cross relaxation with water protons (Yarnykh et al., 2018). MPF mapping is a new technique, which has been histologically validated and shows promise as a tool for accurate evaluation of fine changes in myelin content in fetal brain (Yarnykh and Yuan, 2004).

Magnetization transfer imaging is heavily dependent on experimental conditions, including scanner type and sequence characteristics (Tozer et al., 2003). In addition, due to the interaction with other physical parameters of a two-pool model, such as the longitudinal relaxation rate $\left(R_{1}=1 / T_{1}\right)$, which also correlates with myelin content and is sensitive to various physiological parameters, including iron and calcium levels, axon count and size, the association between MTR and myelin content in tissue is nonlinear (Henkelman et al., 2001). Further, it is argued that MTR is sensitive to macromolecules associated with other cellular components, such as cytoskeleton, and, therefore, a solution given by a two-pool seems to be insufficient to accurately access complex interactions between molecular pools in living tissue (Sled and Pike, 2001).

Throughout development, MTR gradually increases in the posterior-to-anterior direction (Rademacher et al., 1999). Myelination rate is faster in the projection and commissural than in the association fibers, as indicated by lower MTR values in the association areas at first month of age (Rademacher et al., 1999). In the occipital and frontal regions, MTR reaches peak by 13 and 16 months, respectively. MTR in the thalamus, splenium and genu of the CC reaches $95 \%$ of the adult value by 10,18 , and 19 months, respectively (Xydis et al., 2006). Throughout adulthood, MTR in the occipital and temporal regions slightly increases, remaining relatively stable in other areas (Mehta et al., 1995; Armstrong et al., 2004).

Macromolecular proton fraction in the fetal brain (18-38 weeks) is fivefold lower than in adult brain (Yarnykh et al., 2018). In the brainstem, cerebellum, and thalamus it shows positive association with gestational age (Yarnykh et al., 2018).

The values of $R_{1}$, which is principally sensitive to macromolecular fraction and tissue volume (Mezer et al., 2013; Stüber et al., 2014), reach peak between ages 30 and 50 years, and between 70 and 80 values drop substantially to values comparable to 8-year-old values (Yeatman et al., 2014). Over an
80 -year period, changes in $\mathrm{R}_{1}$ follow parabolic curve, indicating a symmetric pattern of age-related changes.

In an adult brain, $R_{1}$ values vary considerably among tracts, and their mature $R_{1}$ and the rate of $R_{1}$ changes are different (Yeatman et al., 2014). For example, the age-related $R_{1}$ growth and decline in in the cingulum and ILF are twice as higher than those of the corticospinal tract and anterior thalamic radiation. Unlike $R_{1}$, the rate of change in diffusivity during development exceeds that of aging, and therefore diffusivity data, particularly MD, do not follow a symmetric parabolic pattern, but instead can be described by a Poisson curve, sharply decreasing throughout childhood and adulthood, and then slowly increasing in the third decade of life (Lebel et al., 2012).

Although indices associated with macromolecular content in tissues seem to provide a more accurate estimate of developmental gain and age-related decline in WM than diffusion metrics, MTI is now mostly used in clinical research, and, to date, there is not enough normative pediatric data to comprehensively describe WM changes throughout development.

\section{g-Ratio}

A new method which allows for the assessment of relative myelination and conduction velocity of an axon is based on the evaluation of the $g$-ratio (i.e., the ratio of the inner to the outer diameter of the myelin sheath; Waxman, 1980). This method has been introduced in 2011 and was applied in full brain WM imaging in 2013 (Campbell et al., 2018). The $g$-ratio is quantitatively expressed as a function of the myelin volume fraction (MVF) and the axon volume fraction (AVF):

$$
g=\sqrt{\frac{1}{1+M V F / A V F}}
$$

Diffusion MRI methods are used to assess the relative AVF by measuring the shape of the displacement distribution of water molecules and evaluation of the intra-axonal volume fraction within the diffusion visible volume (Campbell et al., 2018). The most popular diffusion models, which can be used to estimate the relative size of cellular compartments include neurite orientation density and dispersion imaging (Zhang et al., 2012), composite hindered and restricted model of diffusion (Assaf and Basser, 2005), diffusion basis (Wang et al., 2011), restriction spectrum imaging (White et al., 2013), and other methods described in detail in Campbell et al. (2018).

Myelin volume fraction can be obtained from MT saturation index (Helms et al., 2009; Campbell et al., 2018), quantitative MT imaging parameters (Campbell et al., 2018), MTR (Yarnykh and Yuan, 2004), proton density imaging (Duval et al., 2017), single-point two-pool modeling (Yarnykh, 2012), quantitative multicomponent $\mathrm{T}_{2}$ (Oh et al., 2006) or $\mathrm{T}_{2}{ }^{*}$ (Du et al., 2007) relaxation techniques quantifying the myelin water fraction, macromolecular pool size from quantitative MT (Yarnykh, 2002), and single-point two-pool and inhomogeneous MT (Varma et al., 2015). $g$-ratio shows considerable variation among different fiber pathways and its values are shown to be in agreement with ex vivo histological measures (Ellerbrock and Mohammadi, 2017). However, the accuracy of the $g$-ratio largely relies on 
the calibration procedure, since the models used to assess MVF do not consider a tissue model, as diffusion models do (Campbell et al., 2018). Selection of an appropriate calibration approach significantly influences the obtained $g$-ratio, and, therefore, affects repeatability and comparability of the data (Ellerbrock and Mohammadi, 2017).

Despite the high potential of the $g$-ratio for in vivo estimation of relative myelination and the application of this method in normative and clinical studies of white matter evaluation, it has not been yet introduced into routine research practice, and the data on $g$-ratio changes across development are still limited (Dean et al., 2016).

The developmental trajectory of the myelin $g$-ratio is still unknown. It is expected that $g$-ratio decreases over time, as axon growth rate exceeds speed of myelination. Only a few studies have examined age-related changes in $g$-ratio (Dean et al., 2016; Cercignani et al., 2017). Throughout infancy and early childhood, $g$-ratio logarithmically decreases, reaching theoretically predicted values (Dean et al., 2016). Age-related nearly-linear increase in $g$-ratio from 20 to 76 years of age has been demonstrated with no gender effect identified (Cercignani et al., 2017).

\section{Myelin Mapping}

An alternative method for assessing cortical myelin content, which is highly generalizable across different scanners and pulse sequences, and, thus, allows for the comparison of the data across studies, is based on combining $\mathrm{T}_{1}$-weighted $\left(\mathrm{T}_{1} \mathrm{w}\right)$ and $\mathrm{T}_{2}$ weighted $\left(\mathrm{T}_{2} \mathrm{w}\right)$ MRI to map myelin content (Glasser and Van Essen, 2011). $T_{1}$ and $T_{2}$ relaxation times reflect the interaction between water and macromolecules in tissue (Miot-Noirault et al., 1997). $\mathrm{T}_{1}$ strongly depends on membrane composition (Turner, 2019), particularly on lipid content (i.e., cholesterol and cerebroside levels; Koenig, 1991). Image intensity (e.g., brightness of the $\mathrm{T}_{1} \mathrm{w}$ image) reflects the distribution of myelin-associated macromolecules, whereas $\mathrm{T}_{2}$ relaxation time is associated with the molecular exchange and water diffusion, such that the lower intensity of a $\mathrm{T}_{2} \mathrm{w}$ image corresponds to higher myelin content (Miot-Noirault et al., 1997; Barkovich, 2000).

The $\mathrm{T}_{1} \mathrm{w} / \mathrm{T}_{2} \mathrm{w}$ index makes it possible to quantitatively assess spatial distribution of myelin, to eliminate MR-related image intensity bias, and to increase the contrast to noise ratio (Glasser et al., 2013, 2014). Fast scanning time makes this method valuable for pediatric imaging. However, due to high sensitivity to intensity scale inconsistencies across different datasets, this method requires strict calibration, which is currently a matter of research (Ganzetti et al., 2014).

In summary, various techniques have been used to assess maturation of WM tracts in the human brain. Considerably more research is needed to replicate and validate findings across methodologies, which will lead to optimized scanning protocols for healthy and clinical signatures of WM changes. As the majority of the literature evaluates maturation of WM tracts using DTI, in what follows we focus on this literature to review considerations related with anatomical characteristics of common fiber tracts and results associated to age, gender and cognition.

\section{ANATOMICAL CHARACTERISTICS OF COMMON FIBER TRACTS}

\section{Superior Longitudinal Fasciculus and Arcuate Fasciculus}

A major association WM tract that links the temporoparietal junction and parietal areas with the frontal lobe is the SLF. Three SLF branches connect the ipsilateral frontal and opercular areas to the superior parietal lobe (dorsal subdivision, SLF I), to the angular gyrus (middle branch, SLF II), and to the supramarginal gyrus (ventral subdivision, SLF III). They are comprised of the frontoparietal network of the human brain with the SLF I being symmetric between the two hemispheres, and the SLF II and SLF III demonstrating rightward lateralization (Thiebaut de Schotten et al., 2011a). The AF is the fourth subdivision of the SLF, which links the posterior and middle superior temporal gyrus with the ventrolateral prefrontal cortex and the posterior region of Broca's area (pars opercularis, Brodmann's area 44; Kamali et al., 2014; Webb, 2017). However, some authors suggest that the AF is distinct from the SLF, with the AF connecting the caudal part of the superior temporal area with the dorsal prefrontal region of the cerebral cortex and comprising of the long, anterior, and posterior segments (Schmahmann and Pandya, 2006; Catani and Thiebaut de Schotten, 2012), and the SLF linking frontal and parietal cortical areas (Thiebaut de Schotten et al., 2011b; Forkel et al., 2020), although the anterior segment of the AF and the SLF III overlap (Forkel et al., 2020). The branch of the SLF, which connects the temporal cortex to the inferior parietal cortex (temporoparietal subdivision, SLF TP) is considered as the fifth subcomponent (Catani et al., 2005; Makris et al., 2005; Zhang et al., 2010; Webb, 2017), although it rarely appears in dMRI tractography studies.

\section{Cingulum}

The cingulum is an association WM tract which extends sagittally and connects the orbital frontal regions with the pole, passing along the dorsal surface of the CC down the temporal lobe (Bubb et al., 2018). This bundle is viewed as a part of the limbic system and considered one of the central components of Papez circuit (Papez, 1995), which constitutes bilateral WM pathways between the anterior thalamic nuclei and cingulate cortex, as well as the parahippocampal region and the cingulate cortex. A substantial portion of cingular association fibers that run across the sagittal plane form intracortical connections, linking the medial parts of the frontal, temporal, and parietal lobes (Yakovlev and Locke, 1961; Schmahmann and Pandya, 2006). dMRI tractography reveals three components of the cingulum: subgenual, retrosplenial or supracallosal, and ventral parahippocampal subdivisions (Jones et al., 2013b), which are characterized by different FA values. Based on current anatomical evidence (Vogt et al., 2005; Heilbronner and Haber, 2014), the cingulum can be divided into five subdivisions, including the subgenual, rostral dorsal (anterior cingulate), caudal dorsal (retrosplenial), and temporal (parahippocampal) subcomponents and midcingulate cortical area. 


\section{Inferior Longitudinal Fasciculus}

The ILF is a ventral association WM tract, which connects the anterior temporal lobe, including the superior, middle, inferior temporal, and fusiform gyri, to the lingual, cuneate, lateral-occipital and occipito-polar regions of the occipital cortex (Shinoura et al., 2007; Panesar et al., 2018). Connectivity pattern of the ILF is characterized by a significant leftward-dominance (Panesar et al., 2018) and ventral position.

\section{Inferior Fronto-Occipital Fasciculus}

The IFOF is one of the major association fiber systems, which is recognized as a part of the dorsal visual stream (Schmahmann and Pandya, 2006). It links the occipital cortex, temporo-basal areas, and superior parietal lobe with the frontal regions, passing through the temporal lobe and insula (Martino et al., 2010) and crossing the SLF, AF, ILF, and MdLF (Wu et al., 2016). Highangular-resolution diffusion imaging shows that the IFOF fibers vary in the sites of origin. Most of the fibers originate from the lateral and medial orbital frontal cortices, frontal polar cortex, including the fronto-marginal gyrus and transverse frontopolar gyrus, superior frontal gyrus and inferior frontal gyri, including the pars opercularis, pars triangularis, and pars orbitalis, and from the middle frontal gyrus (Wu et al., 2016). Fibers of the posterior portion of the IFOF terminate in the pericalcarine (cuneus and lingual gyrus) and fusiform gyri and occipital region, including the inferior, middle, and superior occipital lobes, with some fibers terminating in the superior parietal lobe, angular and postcentral gyri (Wu et al., 2016). A comprehensive study by $\mathrm{Wu}$ et al. (2016) suggests that the IFOF has at least five subcomponents characterized by different connectivity patterns, albeit other researchers divide this fasciculus into three (Conner et al., 2018) or two subdivisions (Martino et al., 2010), or consider it as a whole tract (Rofes et al., 2014).

\section{Uncinate Fasciculus}

One of the long-range association bidirectional monosynaptic WM pathways is the UF, which connects the anterior temporal lobes and amygdala to the lateral orbitofrontal cortex and the anterior portion of the prefrontal cortex (Von Der Heide et al., 2013).

Commissural pathways connect two hemispheres and are composed of the CC, the anterior and posterior commissures, the hippocampal commissure, the habenular commissure, and the hypothalamic and cerebellar commissures (Catani and Thiebaut de Schotten, 2012; Gupta, 2017). As it follows from fiber location, commissural pathways play a critical role in the integration and transfer of the information between the motor, perceptual, and cognitive modalities.

Projection fibers pass through the internal capsule, corona radiata, cerebral peduncles, and brainstem connect the cerebral cortex to other subcortical structures, such as spinal cord (SC), deep cerebral nuclei, and brainstem nuclei, forming bidirectional (ascending and descending) connections. Output from the hippocampus to the cortex is formed by the fornix which begins in the hippocampus and passes longitudinally toward the diencephalon and basal forebrain, arching over the thalamus (Catani and Thiebaut de Schotten, 2012; Gupta, 2017).

\section{Middle Longitudinal Fasciculus}

Middle longitudinal fasciculus is an association fiber tract, which was originally identified in the rhesus monkey brain (Schmahmann and Pandya, 2006; Wang et al., 2013), where it links the caudal and rostral portions of the STG, the caudal cingulated gyrus and the middle portion of the parahippocampal gyrus with the upper sector of the STG (Schmahmann and Pandya, 2006).

Neither the classical (Ludwig and Klingler, 1956), nor the contemporary fiber microdissection studies (Wang et al., 2013) have described the anatomical structure of MdLF. The existence of the MdLF has been first documented in a DTI study by Makris et al. (2009). Although DTI does not allow for an accurate tracking of the MdLF origin and destination, especially in voxels containing crossing fibers (Wang et al., 2013), more studies demonstrate successful delineation and differentiation of the MdLF from other ventral and dorsal association fiber pathways (Menjot de Champfleur et al., 2013). Notably, human dMRI tractography and microdissection studies show prominent differences in MdLF anatomy between humans and monkeys. As such, the human MdLF was shown to link the STG with the superior parietal lobule and parietooccipital region, with only minor connections with the angular gyrus, unlike monkey brain (Wang et al., 2013).

\section{Frontal Aslant Tract}

The FAT interconnects the pars opercularis and pars triangularis of the inferior frontal gyrus and the anterior insula with the supplementary motor area (SMA) and pre-SMA (Chernoff et al., 2018; La Corte et al., 2021), and it was first identified in 2007 by Aron et al. (2007) and then described and named in the studies of Catani et al. (2012) and Thiebaut de Schotten et al. (2012). In addition, it was also identified in monkey (Thiebaut de Schotten et al., 2012) and human postmortem dissection studies (Lawes et al., 2008).

Maturation of fiber tracts has been documented in vitro (e.g., Yakovlev and Lecours, 1967). In what follows we focus on in vivo studies that use DTI to evaluate typical development identifying consistencies and inconsistencies in reports related to age, gender and cognitive abilities.

\section{MYELINATION AND AGE}

The process of myelination follows a complex topographical and temporal pattern across development as demonstrated by histological studies (Yakovlev and Lecours, 1967). Changes in myelination are proposed to be synchronized among functionally related fiber networks (Valk and Knaap, 2013). In DTI studies, WM maturation is traditionally examined by assessing the changes in several metrics, including $\mathrm{AD}$ and $\mathrm{FA}$, which increase with age as myelin thickness and axonal diameter and density increase, and $\mathrm{RD}$ and $\mathrm{MD}$, which are known to decrease with age (Tamnes et al., 2018). 
Critically, there is no period in development when WM microstructure in the brain is static. During childhood, adolescence, and early adulthood, WM maturation is fast and dynamic and shows pronounced increase in fiber density and myelination as indicated by an increase in FA and decrease in MD and RD (Schmithorst et al., 2002; Westlye et al., 2010; Lebel et al., 2012; Sexton et al., 2014). During mid-adulthood, WM structure is relatively stable, and in late adulthood, changes in WM accelerate again, reflecting degenerative processes, as indicated by a sharp reduction of FA and an increase in MD and RD (Schmithorst et al., 2002; Lebel et al., 2012).

The speed of myelination in a given region during ontogenesis corresponds to the position of the system in the functional hierarchy. The first site of myelination is the SC. Here, myelination begins at 12-14 weeks of pregnancy (Choi, 1981). Myelination cycle of the motor root fibers is faster and shorter than that of sensory roots. The last site of myelination in adults is intracortical fibers of the cerebral cortex (Yakovlev and Lecours, 1967). Within the period between midgestation and the end of the second postnatal year, this process is characterized by the greatest rate and undergoes the most dramatic changes (Brody et al., 1987; Engelbrecht et al., 1998).

Myelination of the cranial nerve roots terminates early in the first postnatal year. The genu of the CC is myelinated by the 6 th postnatal month. In the frontal, parietal and occipital lobes, the fibers are myelinated by $8-12$ months of postnatal life (Barkovich and Kjos, 1988; Bird et al., 1989; Paus et al., 2001). In the central nervous system, sensory fibers which form the inputs to the thalamus and cerebral cortex myelinate earlier than fibers which participate in the integration of sensory information into movements (Bird et al., 1989; Paus et al., 2001). The longest myelination cycle is characteristic for the commissural and association fibers of the supralimbic zones. It exponentially continues to the years of maturity to senium (Yakovlev and Lecours, 1967). The development of the major fiber pathways from preterm age to the first postnatal age are summarized in Figure 2.

Myelination of the cerebral WM begins in the tenth fetal month. The most rapid growth of the brain occurs during the first 3 years of life (Dekaban, 1978). During childhood, an increase in WM volume exceeds that of the GM (Groeschel et al., 2010). A key common trend in WM development found across the majority of studies is its non-linear pattern across childhood and adolescence, accompanied by an overall increase in FA and a decrease in MD during the first years of life, which is more pronounced during early childhood (Krogsrud et al., 2016; Lebel et al., 2019). Fibers in the left hemisphere show greater maturation rate, and changes in myelination indices demonstrate an overall posterior-to-anterior gradient (Krogsrud et al., 2016). Interestingly, FA in the association tracts reaches its maximum 3-7 years before MD reaches minimum (Lebel et al., 2012). The transition from childhood to adolescence is accompanied by a pronounced increase in FA in the major fiber tracts, including the ILF, SLF, IFOF, fornix, cingulum, UF, and all subdivisions of the CC, indicating an increase in fiber density and myelination. Changes in MD show nonlinear pattern, reaching minimum between the age of 18-41 years and then gradually increasing
(Lebel et al., 2012). Early development is characteristic for the CC and fornix, whose myelination is complete by the age of 20 , whereas pathways which form frontal-temporal connections, including cingulum, UF, and SLF, demonstrate a more protracted maturation cycle, with the cingulum reaching peak FA values after 40 years (Lebel et al., 2012).

Myelination of the CC in children is incomplete (Brown et al., 2005), and with age it continues to mature, as it is one of the critical structures that sustains interhemispheric interaction (Banich and Brown, 2000). Importantly, different subdivisions of the CC demonstrate high degree of variability in size and maturation rates (Giedd et al., 1996). Posterior and mid regions of the CC show greater dependence on age compared to anterior portion of the tract, including the rostrum and genu of the CC that reach adult size in young children, thus exhibiting anterior to posterior gradient of myelination (Giedd et al., 1996). FA in the genu and splenium of the CC reaches peak values at 21 and 25 years, respectively, and in the body of the CC FA reaches maxima at the age of 35 years (Lebel et al., 2012). Critically, the assessed rate and temporal pattern of development of the splenium often vary by the age range of study participants and the fitting method used to model callosal maturation (Lebel et al., 2019).

Findings on age-related changes in other DTI metrics, such as RD, which reflects the rate of water diffusion in the direction perpendicular to the main axis of the axon, show that the most active maturation of association and projection pathways, which maintain cortical and brainstem integration, occurs during adolescence (Johansen-Berg and Behrens, 2009; Kumar et al., 2013). This is concurrent with the behavioral observations of pronounced improvements in reaction time that continue into late adolescence and adulthood (Asato et al., 2010; Arsalidou et al., 2013). Between 10 and 12 years of age, it is estimated that brain weight reaches adult values (Hedman et al., 2012). This period of WM maturation is accompanied by the maturation of critical cognitive functions, such as executive control and interhemispheric communication (Asato et al., 2010). The majority of fiber tracts, including the CC, SLF, ILF, IFOF, UF, and cingulum exhibit nonlinear developmental trajectory, as indicated by quadratic increases in FA and MD (Tamnes et al., 2018).

In the adult brain, the three myeloarchitectonic regions myelinate at different rates (Olson et al., 2015; Williamson and Lyons, 2018). Consistent with in vitro studies, DTI studies demonstrate that myelination of most association tracts continues into the third decade of life in a well-defined order (Olson et al., 2015; Williamson and Lyons, 2018). Also, agerelated decrease in myelination in these fibers is delayed (Lebel et al., 2012). Myelination of the SC is mainly studied in animal and clinical studies (Tu et al., 2013; Antherieu et al., 2019; Mishra et al., 2020). Cervical SC microstructural properties are vulnerable to aging, as FA in this subdivision shows strong negative correlation with age (Agosta et al., 2007) and it dramatically decreases after the age of 55 years (Vedantam et al., 2013). Across the SC subdivisions, FA decreases in rostralcaudal direction, whereas $\mathrm{MD}$ remains relatively constant. The $\mathrm{AD}$ in the cervical subdivision is larger in thoracis and lumbar segments, indicating the presence of large-diameter myelinated 


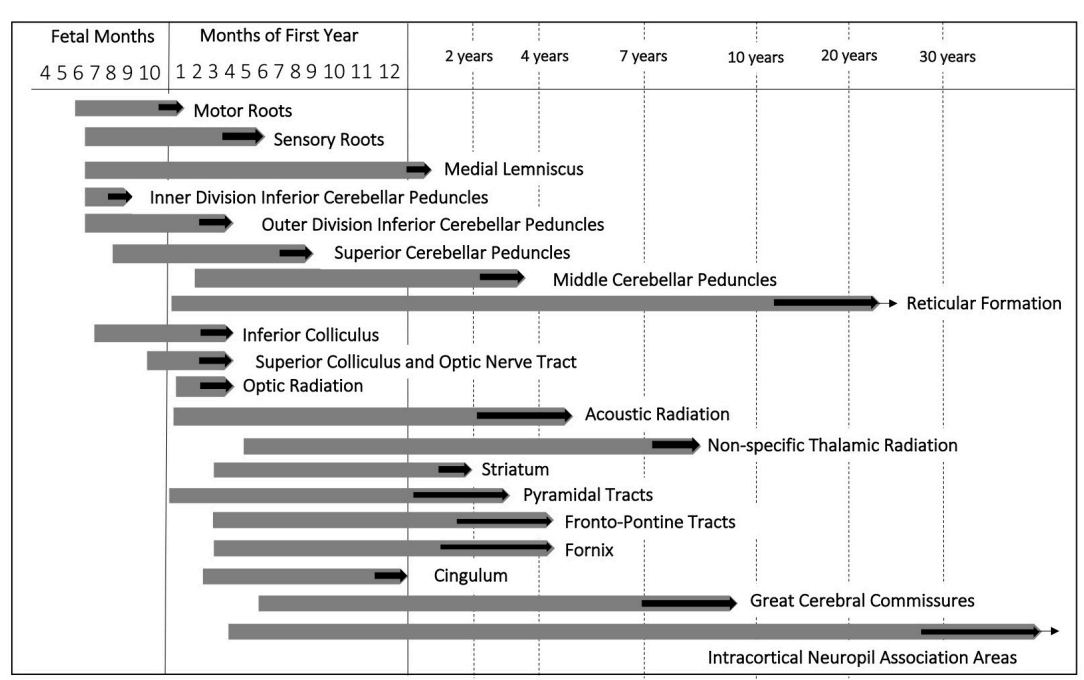

FIGURE 2 | Myelination cycle adapted from Yakovlev and Lecours (1967). Chronological pattern of fiber myelination in the central nervous system. Thick black arrows indicate the approximate age range within which myelination of a given fiber tract terminates. Extending ends of the bars correspond to increasing staining intensity and density of myelinated fibers.

axons (Vedantam et al., 2013). Notably, higher FA in the C2C7 of the cervical SC predicts better performance in visuomotor tracking of precision grip force (Vedantam et al., 2013). The study of Lindberg et al. (2010) demonstrated asymmetry in WM density in the cervical SC: FA was higher in the lateral portion of the SC and on the right side, compared to the medial SC and the left side, respectively. Notably, across all cervical segments, only FA in the medial SC correlated with age, indicating higher sensitivity of this subdivision to aging.

During the transition from childhood (6-11 years) to adolescence (12-16 years), FA, fiber density and length in the SC increase, whereas MD decreases (Alizadeh et al., 2018). Findings on the association between other DTI metrics and age are controversial. Some studies demonstrate an increase in AD and MD with age (Reynolds et al., 2019), whereas others find an age-related decline in diffusivity measures (Singhi et al., 2012; Saksena et al., 2016).

Overall, finding on age-related changes in DTI indices in the SC are limited, and the association between SC myelination and cognitive and motor skill development is yet to be explored.

Critically, review studies that examine normative values across development, recognize that different methodological approaches such as preprocessing, statistical analyses and age group limits contribute to result differences among studies (Tamnes et al., 2018; Lebel et al., 2019). Specifically, they emphasize that linear models may not characterize the dynamic non-linear maturation patterns in myelination that occur across development and identify the need for improved methods (Tamnes et al., 2018; Lebel et al., 2019). Notably, dMRI tractography results are highly dependent on optimization algorithms (Harms et al., 2017). One of the most advanced algorithms is the gradient-free Powell conjugate-direction algorithm, which allows for considerable improvement of run time, parameter fit, and accuracy, whereas the parameter initialization approaches provide better fit for complex models (Harms et al., 2017).

\section{MYELINATION AND GENDER}

Histological methods greatly contributed to the understanding of temporal pattern and regional specificity of the WM, however, data on gender differences in myelination pattern have been mostly obtained using MR-based techniques. Although male and female brains show many similarities, some studies highlight differences between the genders (e.g., Kanaan et al., 2012; Xin et al., 2019), yet others suggest that these differences are driven by environmental and cultural factors (Jäncke, 2018).

The proton density and $\mathrm{T}_{2}$-weighted MRI data corrected for total intracranial volume demonstrate that females exhibit a higher proportion of GM, while males are characterized by a greater percentage of total WM and cerebrospinal fluid (Gur et al., 1999), albeit some studies indicate that these differences are insignificant (Bourisly et al., 2017). Critically, the absolute differences in brain size and regional differences in the proportions of GM and WM between males and females mostly correspond to differences in WM volume (Passe et al., 1997; Allen et al., 2003; Schmithorst et al., 2008). In addition, after correcting for total cranial volume, no significant differences in the volumes of amygdala, hippocampus, and the dorsal prefrontal cortex between men and women are reported, although adult females are found to have significantly greater volume of orbitofrontal cortical region (Gur et al., 2002).

Several MRI studies demonstrate that the pattern of white and gray matter maturation in certain brain regions differs in males and females (De Bellis et al., 2001; den Braber et al., 2013; Ritchie et al., 2018). The total volumes of the cerebral gray and WM and CC show a pronounced sex-age interaction with males exhibiting 
a more significant age-related reduction of the gray matter and an increase in the WM volume and callosal region (De Bellis et al., 2001). According to the results of voxel-based morphometry analysis carried out by Bourisly et al. (2017), WM volume in the frontal, temporal, parietal, occipital, and insular regions of the brain is larger in men compared to women, and only WM of the postcentral gyrus in the right parietal lobe is greater in women compared to men. However, the authors of the study did not control for the total brain volume, although they claim that these differences cannot be explained by an assumption that the brain volume of men is, in general, bigger than that of women.

Data on gender differences in WM in frontal regions are contradictory. A study by Szeszko et al. (2003) reports that adult women have higher FA in the left frontal lobe compared to men. In addition, females exhibit a leftward frontal asymmetry in FA (i.e., higher FA values in the left hemisphere), which correlates with a better comprehension of verbal constructions and memory functioning in women, whereas males show no such asymmetry. These differences may be explained by the fact that maturation of the WM in the frontal lobes continues into late adulthood with a peak at about 45-50 years of age (Bartzokis et al., 2001; Schmithorst et al., 2008). A DTI study by Kanaan et al. (2012) found that adult males have higher FA values in the cerebellum and at the anterior portion of the left SLF, whereas females show greater FA values in the $\mathrm{CC}$, interpreted to contribute to better interhemispheric communication and intellectual performance (Luders et al., 2007). Boys are also characterized by greater FA values in bilateral frontal $\mathrm{WM}$, in the right $\mathrm{AF}$, and in the left parietal and occipito-parietal WM. Interestingly, in the left frontal lobe, boys exhibit a positive and girls show a negative correlation between age and FA. In contrast, FA in the right AF positively correlates with age in girls and negatively in boys. In the right frontal lobe and right occipito-temporo-parietal WM girls exhibit a positive association between FA and age, whereas no significant correlation is found in boys (Schmithorst et al., 2008). An MRI study of healthy children and adolescents (6-17 years; Blanton et al., 2004) shows significant right > left asymmetry in the total cerebral volume, total cerebral WM, and WM of the middle and superior frontal gyri of boys, whereas girls are characterized by the same asymmetry pattern in the total cerebral WM and WM of the superior frontal gyrus. In addition, WM in the left inferior frontal gyrus shows age-dependent increase in boys, but not in girls. The authors speculate that these differences may be associated with gender effects in the development of speech and language lateralization.

The most rapid changes in the developmental pattern of the CC occur during infancy (Schmied et al., 2020) and findings on gender influence on the structure and development of the CC are inconsistent and largely depend on method and age when the microstructure was examined. One of the factors that may cause the discrepancies in findings on gender differences in the CC microstructure and development is the size of the brain: males, on average, are characterized by larger brain size than females, and therefore the size of the CC, if not adjusted for brain size, is also larger in males (Bishop and Wahlsten, 1997). Early data show no differences in the size and shape of the splenium of the $\mathrm{CC}$ between males and females, irrespective of the adjustment for brain size (Bishop and Wahlsten, 1997). However, recent DTI data on callosal area and thickness from infants (6-24 months) adjusted for brain size demonstrate a higher growth rate and size in boys than in girls, although the association between the microstructural characteristics and callosal size exhibits no significant gender differences (Schmied et al., 2020). Sullivan et al. (2010) show a significant gender-related difference in the development of the CC with age and their association with lower performance on cognitive and motor tasks observed in older adults. A DTI study by Schmithorst et al. (2008) shows that girls (mean age $\sim 12$ years) have higher FA in the splenium of the CC than boys. Interestingly, the greater size of the splenium of the CC observed at autopsy was not confirmed by an early MRI study, which, reported a gender-related difference in minimum width of the callosal body and an age-related decline in anteroposterior distance and significant intraindividual variations in callosal size and shape regardless of age or gender (Byne et al., 1988).

Mechanisms that drive gender effects may rely on biological and experiential factors. Early studies suggest that differences in spatial and temporal pattern of myelination in boys and girls may be due to changes in sex steroid hormone levels during critical periods of development, accompanied by a 26 -fold increase in testosterone and a 10-fold increase in estradiol level in males and females, respectively (Ducharme et al., 1976). Although steroid hormones are essential for myelination in both sexes, current research show that age-related changes and gender differences in sensitivity and responsiveness of brain structures to hormonal influences may explain different effect exerted by testosterone and estradiol on male and female brain at different stages of development (Patel et al., 2013).

Estradiol is known to affect hippocampal (Ducharme et al., 1976) and Schwann cell proliferation and differentiation (Chen et al., 2016), regulation of synaptic function, morphology, and plasticity (Smith et al., 2009), modulation of synaptogenesis and remodeling of neuronal circuits (Srivastava and Penzes, 2011; Sellers et al., 2015). Testosterone, in turn, stimulates neuronal growth at early stages of development, and both neuronal growth and myelogenesis at later stages (Stocker et al., 1994).

Normative values for DTI indices may differ across studies. Moreover, different imaging methods may provide different estimates of WM microstructure (Scholz et al., 2009). For example, some DTI studies show that the CC is more myelinated in males than in females (Westerhausen et al., 2003; Shin et al., 2005), whereas $T_{1}$-weighted imaging demonstrates and opposite pattern (Scholz et al., 2009). Maturation pattern in some fiber tracts is still poorly understood due to extant disagreements on the structure of the tracts. Further, a thorough review and meta-analyses of human MRI and postmortem data cautions that gender effects in human brains are largely based on overall brain size and in the few instances that they appear they contribute only to $1 \%$ of variance in structure (Eliot et al., 2021). Therefore, when investigating gender-related differences in WM development and microstructure, it is important to consider differences in total brain size, and to take into account specificity of the method applied and age of the participants, since myelination rate at different developmental stages is different. Moreover, the comparison of the neuroimaging results with the 
post mortem findings may help to clarify the discrepancies across methods. Further research, especially longitudinal studies and meta-analyses that identify overarching patterns in the data, are needed to evaluate effects in WM between the two genders.

\section{MYELINATION AND COGNITIVE ABILITIES IN COMMON FIBER TRACTS}

White matter pathways, which connect distributed brain areas, play a fundamental role in the maintenance of higher-level cognitive functions and are pivotal for cognitive, motor, and behavioral performance. A DTI study by Johansen-Berg et al. (2007) found an association between bimanual coordination and characteristics of WM microstructure in the CC which connects the supplementary motor area and caudal cingulate motor area. Compared to an object recognition task (Baird et al., 2005), high FA in the splenium and genu of the CC were associated with shorter and longer reaction times, respectively. The task on object recognition was presented from unusual viewpoints, which required transduction of the information from the right parietal cortex to the left inferior parietal cortex. Since myelin sheath increases signal transduction and its formation continues during adulthood, brain myelination plays an important role in inhibitory control and executive functions in children and adolescents, thus underlying healthy maturation (Bartzokis and Altshuler, 2005).

Research suggests that greater axonal volume and myelination in particular brain regions, including the parietal cortical areas, left superior and posterior corona radiata, and body of the CC contribute to better performance on auditory working memory tasks, such as letter-number sequencing, by sustaining higher speed of information processing (Chung et al., 2018). Tamnes et al. (2013) suggest that myelination and maturation of synaptic connections in fronto-parietal brain networks during adolescence is implicated in greater specialization and processing efficiency, which, in turn, mediates the development of executive functions in children and adolescents. As shown in a DTI study by Tuch et al. (2005), the correlation between microstructural measurements of WM in the right projection and association pathways, such as the right optic radiation, right posterior thalamus, and right medial precuneus, with visual self-paced choice task performance, reflects the fundamental role of myelination in these regions for maintenance of visuospatial attention. Notably, microstructural characteristics of WM pathways, including FA and neurite density, show a positive correlation with mathematical performance in 13year-olds (Collins et al., 2019). Myelination indices in the left superior corona radiata correlate with numerical operations and mathematical reasoning, whereas WM microstructure characteristics in the left ILF show associations with numerical operations specifically (van Eimeren et al., 2008).

Myelin is a principal component of WM and it accounts for approximately $50-60 \%$ of the WM dry weight (Snaidero and Simons, 2014). Stimulation of oligodendrocyte precursor cells results in their transformation to mature oligodendrocytes (Snaidero et al., 2014). Although the majority of tracts are myelinated by adulthood, several WM areas, such as those in prefrontal regions and optic radiation, reach peak of myelination between the third and fourth decades of life (Giedd et al., 2015; Turner, 2019). Some suggest that myelination is mainly driven by neuronal activity (i.e., generation and passage of action potentials along the axonal membrane), which can trigger an increase in axonal diameter (Costa et al., 2018). Once the axonal diameter exceeds $0.5 \mathrm{mcm}$ (Nave and Werner, 2014) and the number of action potentials generated along the unmyelinated axonal membrane is sufficient to stimulate conversion of oligodendrocyte precursor cells to mature cells, oligodendrocytes wrap nearby axons by myelin layers (Snaidero et al., 2014).

In mature axons, fine tuning of myelination speed and modulation of signal transmission are controlled by changes in myelin thickness (Waxman, 1975; Campbell et al., 2018) and the length of myelinated axonal regions (Rushton, 1951; Brill et al., 1977), axonal diameter (Chéreau et al., 2017), and topographical organization of the nodes of Ranvier within the axons (ArancibiaCárcamo et al., 2017). Particularly, along with the number of myelin layers and internode length, node organization to a large extent determines conduction speed along myelinated axons, and modulation of the lengths of the nodes of Ranvier serves an efficient mechanism to control signal conduction (Arancibia-Cárcamo et al., 2017).

It is proposed that myelination can be activity-dependent (i.e., depend on electrical activity of the axon and various molecular mediators synthesized and released in response to electrical events), and activity-independent (i.e., regulated by oligodendrocytes and independent from on the axonal electrical activity; De Faria et al., 2019 for review). An intriguing work by Bechler et al. (2018) discusses a "smart wiring" model of myelination, which includes two phases - intrinsic and adaptive, and suggests possible mechanisms by which active axons may become more myelinated and how brain circuits may be modified in response to learning and new experience. Bechler also discusses adaptive myelination, which is provided by several properties of oligodendrocytes. Oligodendrocytes in the central nervous system are generated from special progenitor cells, which actively divide throughout the whole life (Rivers et al., 2008; Hughes et al., 2013). Moreover, proliferation and differentiation of oligodendrocyte precursor cells was demonstrated to improve motor learning and learning new motor skills, which in turn, changes the structure of the WM containing many lateborn oligodendrocytes (McKenzie et al., 2014). Overall, brain myelination appears to be adaptive, and it may change in response to learning and new experience that relates to fiber tracts that connect brain areas associated with various cognitive, affective and motor functions.

Therefore, research supports that increasing neuronal activity, which accompanies brain maturation and learning is the main driving force for dynamic myelination and regulation of network organization throughout lifespan (Hill et al., 2018; Hughes et al., 2018; Stedehouder et al., 2018). By modulating oligodendrocyte precursor cell proliferation and oligodendrocyte differentiation, increased neuronal activity ensures efficient signal transmission, fine-tuning of conduction speed, and the maintenance of circuit function. Myelin growth, in turn, promotes an additional 
increase in conduction velocity, thus strengthening the existing connections (Fields, 2015; Almeida and Lyons, 2017). This is consistent with developmental cognitive theory that proposes neuropsychological mechanisms that underlie these changes. Specifically, the Theory of Constructive Operators (PascualLeone, 1970; Arsalidou et al., 2019; Pascual-Leone and Johnson, 2021) proposes that operators (i.e., content-free general resources represented by functional-structural constrains) regulate schemes (i.e., information bearers represented by cell assemblies and networks) that produce a mental or action output when following the principle of schematic overdetermination of performance (i.e., following a winner-takes-all approach). This framework allows for ontogenetic, biological influences to drive maturation (i.e., relate more to operators) as well as experiential opportunity to drive learning (i.e., relate more to schemes). As we overview below, structure-function relations may be supported by mechanisms associated with biological maturation and experiential learning.

\section{Corpus Callosum}

The largest fiber tract in the brain is the CC. It consists of more than 200 million axons and plays a key role in integration and interhemispheric transfer of information (Fitsiori et al., 2011). Malformation of the CC results in deficits in higherlevel cognitive functions and social communication (Paul et al., 2014), as well as in a slower rate of cognitive processing, impaired language function (Banich and Brown, 2000; Paul et al., 2003), abstract reasoning, and concept formation (Brown and Paul, 2000). Incomplete myelination of the CC in 6-7-year-old children is associated with behavioral abnormalities and results in impaired transfer of complex visuomotor skills learned by the dominant hand from one hemisphere to another (Chicoine et al., 2000). The observation that myelination of the anterior portion of the CC may be induced by working memory training suggests plasticity of WM microstructure in this region (Takeuchi et al., 2010). Reduced integrity of the CC, in turn, may lead to working memory deficits. In particular, reduced integrity in the callosal subregions, which connect anterior and posterior parietal cortical areas, is associated with lower performance in verbal working memory tasks, and higher RD in the portion of the CC, which links anterior regions with posterior and temporal cortical areas, predicts lower performance in visuospatial working memory task (Treble et al., 2013). Interestingly, the length of the CC shows negative correlation with nonverbal abilities (Schmied et al., 2020). Together with the cingulum, the CC often shows correlations with functional measures in neurological groups (Forkel et al., 2020).

\section{Cingulum}

A number of DTI studies show that the cingulum, especially its dorsal portion, is implicated in cognitive control and various executive functions, including shifting and inhibition (Kantarci et al., 2011; Bettcher et al., 2016), updating and working memory (Charlton et al., 2010; Kantarci et al., 2011; Bettcher et al., 2016), processing speed (Takahashi et al., 2010; Bettcher et al., 2016), and sustained attention (Takahashi et al., 2010). In particular, FA in the right cingulum correlates with task performance on the Continuous Performance Test, which measures sustained attention (Takahashi et al., 2010). MD in the bilateral cingulum shows gender integrations and is associated with internalizing behavior in children and adolescents, which is more pronounced in females (Andre et al., 2020). Interestingly, FA in the left cingulum, as well as in the left UF, shows strong age-behavior interaction (Andre et al., 2020). Microstructural characteristics in the posterior cingulum also demonstrate a strong association with language (category fluency and naming to confrontation) and visual-spatial functions, whereas FA in both posterior and anterior portions of the tract are implicated in attention, executive functions, and memory assessed by free recall retention task performance and auditory verbal learning test (Kantarci et al., 2011). The strongest evidence about the contribution of the cingulum to episodic and verbal memory functions are demonstrated in clinical samples, including Alzheimer's disease (Lin et al., 2014; Kantarci et al., 2017), amnestic mild cognitive impairment (Lin et al., 2014; Yu et al., 2017), cerebral small vessel disease (van der Holst et al., 2013), and acute mild traumatic brain injury (Wu et al., 2010). Together with the AF and UF, the microstructural properties of the cingulum are often reported to be strongly associated with psychiatric symptoms (Forkel et al., 2020).

An intriguing article by Bathelt et al. (2019) describes the use of a data-driven community-clustering algorithm to analyze differences in WM microstructure in children and adolescents (mean age $\sim 11$ ), which identified two subgroups characterized by a prominent difference in FA in bilateral cingulum. Critically, these two brain types differed in cognitive abilities with the higher FA group exhibiting better performance in working memory and long-term memory tasks, fluid intelligence, and vocabulary. Long fibers in the dorsal cingulum run in parallel with the long-range fibers in the SLF, which, in turn, extent more laterality to the AF in each hemisphere.

\section{Superior Longitudinal Fasciculus and Arcuate Fasciculus}

Diffusion tensor imaging studies show that the right SLF is associated with various complex cognitive functions, including attention (Frye et al., 2010) and visuospatial abilities (Hoeft et al., 2007), whereas left SLF plays a fundamental role in language (Dick and Tremblay, 2012) and reading skills (Frye et al., 2010). In adolescents, bilateral SLF is involved in verbal working memory and verbal fluency (Peters et al., 2012). It is proposed that the SLF consists of five branches (Kamali et al., 2014). The SLF I is involved in the regulation of motor behavior and the initiation of motor activity. In addition, it was suggested to transfer somatosensory and kinesthetic information about location of body parts, including trunk and limbs, to the frontal regions of the brain (Schmahmann and Pandya, 2006). The SLF II sustains visual perception and it is a part of the circuit involved in visual awareness and the maintenance of attention. The SLF II fibers connecting the prefrontal cortex with the posterior parietal regions are implicated in regulation of the focusing of attention (Petrides and Pandya, 2002). The SLF III was found to convey higher-level somatosensory information and 
to be involved in gestural communication (Petrides and Pandya, 2002). The dorsal pathway through the lateral SLF supports articulatory function, phonological working memory (Duffau, 2019), perception and production of speech (Indefrey and Levelt, 2004; Hickok and Poeppel, 2007). The AF is implicated in intelligence, reasoning abilities (Lebel and Beaulieu, 2009), and language processing (Geschwind, 1970), playing a critical role in auditory to motor connection (Webb, 2017) and semantic and phonological processes associated with visual information (Zemmoura et al., 2015). The indicators of WM microstructure in the left SLF demonstrate a negative association with inhibitory control, whereas in the left AF they correlate with intelligence tests and attention (Urger et al., 2015). Therefore, findings from the neuroimaging studies demonstrate a critical role of the SLF and $\mathrm{AF}$ in neurophysiological abilities and cognitive function.

\section{Inferior Longitudinal Fasciculus}

The ILF (Shinoura et al., 2007), together with the MdLF (Saur et al., 2008) and UF (Papagno et al., 2011), comprises the ventral stream of language processing and was found to sustain speech comprehension and general semantic processes (Rizio and Diaz, 2016). A proposed role of the ILF in semantic functionality (Mandonnet et al., 2007) is supported by neuroimaging data obtained in the study by Shin et al. (2019), which demonstrates the role of the ILF in language comprehension. The ILF connectivity pattern was also shown to be leftward-lateralized and greater fiber density and myelination in this region were found to be associated with better semantic processing. Moreover, the ILF is known to be implicated in object (Ortibus et al., 2012) and face (Taddei et al., 2012) recognition. Some earlier studies show that the peak of myelination in the ILF occurs at 11 years of age (Lebel et al., 2008), whereas recent findings demonstrate that the maturation of this fiber tract continues until age of 24 years (Lebel and Beaulieu, 2011; Lebel and Deoni, 2018). Notably, age has a great impact on lateral tracts in dorsal areas of the brain, including the SLF, whereas the inferior regions are less affected by aging (Sullivan et al., 2010). The ILF, together with the AF, demonstrates strong functional correlations with cognitive measure in healthy individuals (Forkel et al., 2020).

\section{Inferior Fronto-Occipital Fasciculus}

Diffusion MRI tractography studies suggest the role of the IFOF in semantic and visual processing and attention (Catani and Thiebaut de Schotten, 2008; Leng et al., 2016). In particular, bilateral IFOF (especially in the left frontal and right occipital parts) is implicated in the maintenance of executive functions, whereas the parietal and insular parts of the left IFOF are involved in alerting (Leng et al., 2016). Higher FA in the IFOF predicts better reading scores (Lebel et al., 2013), and FA in the left IFOF is related to better orthographic processing (Vandermosten et al., 2012). In addition, the IFOF plays a crucial role in multimodal semantic processes, including naming and nonverbal semantic associations (Gil-Robles et al., 2013; Han et al., 2013; Moritz-Gasser et al., 2013; Rollans et al., 2017), constituting a bilateral network, which sustains non-verbal semantic system (Herbet et al., 2017). Moreover, microstructural properties of the IFOF predict object working memory task performance and therefore underlie nonspatial working memory processing (Walsh et al., 2011). However, the anatomical features of the IFOF are still a matter of discussion and there is still no consensus regarding the mechanisms and the degree to which anatomical and microstructural properties of this bundle can vary in healthy brains.

\section{Uncinate Fasciculus}

A DTI study by Sato et al. (2012) demonstrates the association between DTI metrics in the UF and visual memory task performance. Studies of mild cognitive impairment and psychopathology support a critical role of the UF in higher-order cognitive functions, including attention, spatial memory, and emotion recognition (Fujie et al., 2008; Hiyoshi-Taniguchi et al., 2015; Olson et al., 2015; Singh et al., 2016). The involvement of the UF in visual associative learning was found in the DTI study by Thomas et al. (2012), who demonstrated a strong positive association between learning rate and microstructural properties of the left UF, suggesting a critical role of the UF in information retrieval. Other DTI studies show strong relations among microstructural characteristics of this WM tract in the left (McDonald et al., 2008; Niogi et al., 2008) and right (Fink et al., 2010) hemispheres and auditory-verbal processes, but not visual memory in adults (McDonald et al., 2008; Niogi et al., 2008), children, and adolescents (Mabbott et al., 2009). The left and right UF may sustain different neurophysiological processes. As such, Thomas et al. (2015) suggest that the left UF contributes to rapid learning of conditional visual-visual associations, whereas the right UF may mediate immediate retrieval of these associations.

\section{Middle Longitudinal Fasciculus}

Microdissection data on spatial organization of the MdLF point at its role in auditory comprehension as a part of the dorsal auditory stream (Wang et al., 2013). Saur et al. $(2008,2010)$ found that MdLF, together with the extreme capsule, connects cortical regions involved in comprehension of meaningful compared to non-meaningful sentences. Although the functional role of the MdLF is still unknown, some suggest that its anatomy relates to a role in language (Schmahmann and Pandya, 2006; Makris et al., 2009). However, electrical stimulation and resection of this fiber tract in the language dominant hemisphere of a patient with brain tumor did not cause language deficits (De Witt Hamer et al., 2011). Further, as evidence by DTI studies, FA in the MdLF, as well as the tract volume, does not display hemispheric asymmetry, as expected for language-related tracts (De Witt Hamer et al., 2011; Wang et al., 2013). More research is needed to understand the functional role of this fascinating fiber tract.

\section{Frontal Aslant Tract}

Neurosurgical studies suggest the role of FAT in speech planning (Chernoff et al., 2018) and initiation (Fujii et al., 2015), and it is hypothesized to subserve a link between sentence planning and lexical access (Chernoff et al., 2018). Moreover, FAT was recently identified as a part of a motor language stream involved 
in speech production (Dick et al., 2013). The role of FAT in language can be inferred from its leftward lateralization in right-handed individuals, similar to other language-related fiber systems (Catani et al., 2012). Interestingly, intraoperative direct current stimulation of the left FAT leads to shuttering (Kemerdere et al., 2016) and speech deficits (Vassal et al., 2014). Damage to this fiber tract leads to verbal fluency, but not grammar deficits in primary progressive aphasia (Catani et al., 2013). Notably, DTI studies suggest that individual microstructural characteristics of FAT maintain kinematics and visuomotor processes, and are involved in visually guided hand movements, such as grasping (Budisavljevic et al., 2017). In older adults, DTI characteristics of FAT, together with the left SLF, predict working memory performance and are hypothesized to be involved in the maintenance, attending, and manipulation of language-related information (Rizio and Diaz, 2016; Varriano et al., 2018). Due to limited data on the association between FAT microstructure and cognitive function, the role of this fiber pathway is mainly evidenced from studies of functional role of the cortical regions it connects. As such, the role of the right IFG and pre-SMA/SMA in inhibitory control may indicate the involvement of the FAT connecting these regions in executive functions (Dick et al., 2019). There are many questions that remain to be answered on the intricate relation of mental function to structural properties of FAT that link psychology, biology and neuroscience.

\section{CONCLUSION}

Biological development is associated with maturation of WM pathways which connect distant and proximal brain regions and are critical components of cognitive processing. Our review suggests that myelination follows a complex developmental

\section{REFERENCES}

Agosta, F., Laganà, M., Valsasina, P., Sala, S., Dall'Occhio, L., Sormani, M. P., et al. (2007). Evi-dence for cervical cord tissue disorganisation with aging by diffusion tensor MRI. Neuroimage 36, 728-735. doi: 10.1016/j.neuroimage. 2007.03.048

Alexander, A. L., Lee, J. E., Lazar, M., and Field, A. S. (2007). Diffusion tensor imaging of the brain. Neurotherapeutics 4, 316-329. doi: 10.1016/j.nurt.2007. 05.011

Alizadeh, M., Fisher, J., Saksena, S., Sultan, Y., Conklin, C. J., Middleton, D. M., et al. (2018). Age related diffusion and tractography changes in typically developing pediatric cervical and thoracic spinal cord. Neuroimage Clin. 18, 784-792. doi: 10.1016/j.nicl.2018.03.014

Allen, J. S., Damasio, H., Grabowski, T. J., Bruss, J., and Zhang, W. (2003). Sexual dimorphism and asymmetries in the gray-white composition of the human cerebrum. Neuroimage 18, 880-894. doi: 10.1016/s1053-8119(03)00034-x

Almeida, R. G., and Lyons, D. A. (2017). On myelinated axon plasticity and neuronal circuit formation and function. J. Neurosci. 37, 10023-10034. doi: 10.1523/JNEUROSCI.3185-16.2017

Andre, Q. R., Geeraert, B. L., and Lebel, C. (2020). Brain structure and internalizing and externalizing behavior in typically developing children and adolescents. Brain Struct. Funct. 225, 1369-1378. doi: 10.1007/s00429-019-01973-y

Antherieu, P., Levy, R., De Saint Denis, T., Lohkamp, L., Paternoster, G., Di Rocco, F., et al. (2019). Diffusion tensor imaging (DTI) and Tractography of the spinal cord in pediatric population with spinal lipomas: trajectory that may vary by age, fiber tract and hemisphere. Effects of gender were also identified, although differences may be confounded by methodological factors (e.g., not controlling for brain volume) or social and learning opportunities. Although different studies use various methodological approaches and it is a challenge to aggregate data, future studies should examine overarching pattern across studies using meta-analytical approaches. The study of the individual differences in cerebral WM myelination is very important for developmental biology and neuroscience. Practically, understanding how and when myelination changes and its relation to cognitive performance can inform education practice and clinical interventions.

\section{AUTHOR CONTRIBUTIONS}

IB and MA conceptualized the project and revised and finalized the manuscript. IB wrote the first draft. MA edited versions of the manuscript. Both authors contributed to the article and approved the submitted version.

\section{FUNDING}

The results of sections 1-3 (1 - Introduction; 2 - Methods for assessing myelination; 3 - Anatomical characteristics of common fiber tracts) were prepared within the framework of the Russian Science Foundation grant (project No.17-18-01047). Sections 4-7 (4 - Myelination and age; 5 - Myelination and gender; 6 - Myelination and cognitive abilities in common fiber tracts; 7 - Conclusion) were prepared within the Russian Foundation for Basic Research grant (project No. 19-313-51010).

preliminary study. Childs Nerv. Syst. 35, 129-137. doi: 10.1007/s00381-0183935-2

Arancibia-Cárcamo, I. L., Ford, M. C., Cossell, L., Ishida, K., Tohyama, K., and Attwell, D. (2017). Node of Ranvier length as a potential regulator of myelinated axon conduction speed. eLife 6:e23329. doi: 10.7554/eLife.23329

Armstrong, C. L., Traipe, E., Hunter, J. V., Haselgrove, J. C., Ledakis, G. E., Tallent, E. M., et al. (2004). Age-related, regional, hemispheric, and mediallateral differences in myelin integrity in vivo in the normal adult brain. AJNR Am. J. Neuroradiol. 25, 977-984.

Aron, A. R., Behrens, T. E., Smith, S., Frank, M. J., and Poldrack, R. A. (2007). Triangulating a cog-nitive control network using diffusion-weighted magnetic resonance imaging (MRI) and functional MRI. J. Neurosci. 27, 3743-3752. doi: 10.1523/JNEUROSCI.0519-07.2007

Arsalidou, M., Agostino, A., Maxwell, S., and Taylor, M. J. (2013). I can read these colors." orthographic manipulations and the development of the color-word stroop. Front. Psychol. 3:594. doi: 10.3389/fpsyg.2012.00594

Arsalidou, M., Pascual-Leone, J., Johnson, J., and Kotova, T. (2019). The constructive operators of the working mind: a developmental account of mental-attentional capacity. Russian J. Cogn. Sci. 6, 44-70.

Arshad, M., Stanley, J. A., and Raz, N. (2016). Adult age differences in subcortical myelin content are consistent with protracted myelination and unrelated to diffusion tensor imaging indices. NeuroImage 143, 26-39. doi: 10.1016/j. neuroimage.2016.08.047

Asato, M. R., Terwilliger, R., Woo, J., and Luna, B. (2010). WM development in adolescence: a DTI study. Cereb. Cortex 20, 2122-2131. doi: 10.1093/cercor/ bhp282 
Aso, Y., Arita, Y., Miura, Y., Iwao, S., Sumi, K., Nakamichi, A., et al. (2017). Relationship between WM lesions and cognitive function in subjects with mild cognitive impairment. J. Neurol. Sci. 381:317. doi: 10.1016/j.jns.2017.08.898

Assaf, Y., and Basser, P. J. (2005). Composite hindered and restricted model of diffusion (CHARMED) MR imaging of the human brain. NeuroImage 27, 48-58. doi: 10.1016/j.neuroimage.2005.03.042

Baillarger, J. (1840). Recherches sur la structure de la couche corticale des circonvolutions du cerveau. Mem. Acad. R. Med. 8, 149-183.

Baird, A. A., Colvin, M. K., VanHorn, J. D., Inati, S., and Gazzaniga, M. S. (2005). Functional connectivity: integrating behavioral, diffusion tensor imaging, and functional magnetic resonance imaging data sets. J. Cogn. Neurosci. 17, 687693. doi: 10.1162/0898929053467569

Banich, M. T., and Brown, W. S. (2000). A life-span perspective on interaction between the cerebral hemispheres. Dev. Neuropsychol. 18, 1-10. doi: 10.1207/ S15326942DN1801_1

Barkovich, A. J. (2000). Concepts of myelin and myelination in neuroradiology. AJNR Am. J. Neuroradiol. 21, 1099-1109.

Barkovich, A. J., and Kjos, B. O. (1988). Normal postnatal development of the Corpus Callosum as demonstrated by MR imaging. AJNR. Am. J. Neuroradiol. 9, 487-491.

Bartzokis, G., and Altshuler, L. (2005). Reduced intracortical myelination in schizophrenia. Am. J. Psychiatry 162, 1229-1230. doi: 10.1176/appi.ajp.162.6. 1229-a

Bartzokis, G., Beckson, M., Lu, P. H., Nuechterlein, K. H., Edwards, N., and Mintz, J. (2001). Age-related changes in frontal and temporal lobe volumes in men: a magnetic resonance imaging study. Arch. Gen. Psychiatry 58, 461-465. doi: 10.1001/archpsyc.58.5.461

Basser, P. J., and Jones, D. K. (2002). Diffusion-tensor MRI: theory, experimental design and data analysis - a technical review. NMR Biomed. 15, 456-467. doi: $10.1002 / \mathrm{nbm} .783$

Basser, P. J., Mattiello, J., and LeBihan, D. (1994). MR diffusion tensor spectroscopy and imaging. Biophys. J. 66, 259-267. doi: 10.1016/S0006-3495(94)80775-1

Basser, P. J., Pajevic, S., Pierpaoli, C., Duda, J., and Aldroubi, A. (2000). In vivo fiber tractography using DT-MRI data. Magn. Reson. Med. 44, 625-632. doi: 10.1002/1522-2594(200010)44:4<625::aid-mrm17<3.0.co;2-o

Basser, P. J., and Pierpaoli, C. (1996). Microstructural and physiological features of tissues elucidated by quantitative-diffusion-tensor MRI. J. Magn. Reson. B 111, 209-219. doi: 10.1006/jmrb.1996.0086

Bathelt, J., Johnson, A., Zhang, M., and Astle, D. E. (2019). The cingulum as a marker of individual differences in neurocognitive development. Sci. Rep. 9:2281. doi: 10.1038/s41598-019-38894-z

Beaulieu, C. (2002). The basis of anisotropic water diffusion in the nervous system - a technical review. NMR Biomed. 15, 435-455. doi: 10.1002/nbm.782

Beaulieu, C. (2014). “The biological basis of diffusion anisotropy," in Diffusion MRI, Second Edn, Chap. 8, eds H. Johansen-Berg and T. E. J. Behrens (San Diego: Academic Press), 155-183. doi: 10.1016/B978-0-12-396460-1.00008-1

Bechler, M. E., Swire, M., and Ffrench-Constant, C. (2018). Intrinsic and adaptive myelination-A sequential mechanism for smart wiring in the brain. Dev. Neurobiol. 78, 68-79. doi: 10.1002/dneu.22518

Bettcher, B. M., Mungas, D., Patel, N., Elofson, J., Dutt, S., Wynn, M., et al. (2016). Neuroanatomical substrates of executive functions: beyond prefrontal structures. Neuropsychologia 85, 100-109. doi: 10.1016/j.neuropsychologia. 2016.03.001

Billiet, T., Vandenbulcke, M., Mädler, B., Peeters, R., Dhollander, T., Zhang, H., et al. (2015). Age-related microstructural differences quantified using myelin water imaging and advanced diffusion MRI. Neurobiol. Aging. 36, 2107-2121. doi: 10.1016/j.neurobiolaging.2015.02.029

Bird, C. R., Hedberg, M., Drayer, B. P., Keller, P. J., Flom, R. A., and Hodak, J. A. (1989). MR assessment of myelination in infants and children: usefulness of marker sites. AJNR. Am. J. Neuroradiol. 10, 731-740.

Bishop, K. M., and Wahlsten, D. (1997). Sex differences in the human CC: myth or reality? Neurosci. Biobehav. Rev. 21, 581-601. doi: 10.1016/s0149-7634(96) 00049-8

Blanton, R. E., Levitt, J. G., Peterson, J. R., Fadale, D., Sporty, M. L., Lee, M., et al. (2004). Gender differences in the left inferior frontal gyrus in normal children. Neuroimage 22, 626-636. doi: 10.1016/j.neuroimage.2004.01.010

Bodhireddy, S. R., Lyman, W. D., Rashbaum, W. K., and Weidenheim, K. M. (1994). Immunohistochemical detection of myelin basic protein is a sensitive marker of myelination in second trimester human fetal spinal cord. J. Neuropathol. Exp. Neurol. 53, 144-149. doi: 10.1097/00005072-19940300000005

Bourisly, A. K., Gejo, G., Hayat, A. A., Alsarraf, L., Dashti, F. M., and Di Paola, M. (2017). WM sexual dimorphism of the adult human brain. Transl. Neurosci. 8, 49-53. doi: 10.1515/tnsci-2017-0009

Brill, M. H., Waxman, S. G., Moore, J. W., and Joyner, R. W. (1977). Conduction velocity and spike configuration in myelinated fibres: computed dependence on internode distance. J. Neurol. Neurosurg. Psychiatry 40, 769-774. doi: 10.1136/ jnnp.40.8.769

Brody, B. A., Kinney, H. C., Kloman, A. S., and Gilles, F. H. (1987). Sequence of central nervous system myelination in human infancy. I. An autopsy study of myelination. J. Neuropathol. Exp. Neurol. 46, 283-301. doi: 10.1097/00005072198705000-00005

Brown, W. S., and Paul, L. K. (2000). Cognitive and psychosocial deficits in agenesis of the Corpus Callosum with normal intelligence. Cogn. Neuropsychiatry 5, 135-157. doi: 10.1080/135468000395781

Brown, W. S., Symingtion, M., VanLancker-Sidtis, D., Dietrich, R., and Paul, L. K. (2005). Paralinguistic processing in children with callosal agenesis: emergence of neurolinguistic deficits. Brain Lang. 93, 135-139. doi: 10.1016/j.bandl.2004. 09.003

Bubb, E. J., Metzler-Baddeley, C., and Aggleton, J. P. (2018). The cingulum bundle: anatomy, function, and dysfunction. Neurosci. Biobehav. Rev. 92, 104-127. doi: 10.1016/j.neubiorev.2018.05.008

Budisavljevic, S., Dell'Acqua, F., Djordjilovic, V., Miotto, D., Motta, R., and Castiello, U. (2017). The role of the frontal aslant tract and premotor connections in visually guided hand movements. Neuroimage 146, 419-428. doi: 10.1016/j.neuroimage.2016.10.051

Byne, W., Bleier, R., and Houston, L. (1988). Variations in human Corpus Callosum do not predict gender: a study using magnetic resonance imaging. Behav. Neurosci. 102, 222-227. doi: 10.1037//0735-7044.102.2.222

Campbell, J. S. W., Leppert, I. R., Narayanan, S., Boudreau, M., Duval, T., CohenAdad, J., et al. (2018). Promise and pitfalls of g-ratio estimation with MRI. NeuroImage 182, 80-96. doi: 10.1016/j.neuroimage.2017.08.038

Catani, M., Dell'acqua, F., Vergani, F., Malik, F., Hodge, H., Roy, P., et al. (2012). Short frontal lobe connections of the human brain. Cortex 48, 273-291. doi: 10.1016/j.cortex.2011.12.001

Catani, M., Jones, D. K., and Ffytche, D. H. (2005). Perisylvian language networks of the human brain. Ann. Neurol. 57, 8-16. doi: 10.1002/ana.20319

Catani, M., Mesulam, M. M., Jakobsen, E., Malik, F., Martersteck, A., Wieneke, C., et al. (2013). A novel frontal pathway underlies verbal fluency in primary progressive aphasia. Brain 136, 2619-2628. doi: 10.1093/brain/awt163

Catani, M., and Thiebaut de Schotten, M. (2008). A diffusion tensor imaging tractography atlas for virtual in vivo dissections. Cortex 44, 1105-1132. doi: 10.1016/j.cortex.2008.05.004

Catani, M., and Thiebaut de Schotten, M. (2012). Atlas of Human Brain Connections. Oxford: Oxford University Press.

Cercignani, M., Giulietti, G., Dowell, N. G., Gabel, M., Broad, R., Leigh, P. N., et al. (2017). Characterizing axonal myelination within the healthy population: a tract-by-tract mapping of effects of age and gender on the fiber g-ratio. Neurobiol. Aging. 49, 109-118. doi: 10.1016/j.neurobiolaging.2016.09.016

Chang, Y.-S., Gratiot, M., Owen, J. P., Brandes-Aitken, A., Desai, S. S., Hill, S. S., et al. (2016). WM microstructure is associated with auditory and tactile processing in children with and without sensory processing disorder. Front. Neuroanat. 9:169. doi: 10.3389/fnana.2015.00169

Charlton, R. A., Barrick, T. R., Lawes, I. N. C., Markus, H. S., and Morris, R. G. (2010). WM pathways associated with working memory in normal aging. Cortex 46, 474-489. doi: 10.1016/j.cortex.2009.07.005

Chen, Y., Guo, W., Xu, L., Li, W., Cheng, M., Hu, Y., et al. (2016). 17 $\beta$-estradiol promotes schwann cell proliferation and differentiation, accelerating early remyelination in a mouse peripheral nerve injury model. BioMed Res. Int. 2016:e7891202. doi: 10.1155/2016/7891202

Chéreau, R., Saraceno, G. E., Angibaud, J., Cattaert, D., and Nägerl, U. V. (2017). Superresolution imaging reveals activity-dependent plasticity of axon 
morphology linked to changes in action potential conduction velocity. PNAS 114, 1401-1406. doi: 10.1073/pnas.1607541114

Chernoff, B. L., Teghipco, A., Garcea, F. E., Sims, M. H., Paul, D. A., Tivarus, M. E., et al. (2018). A role for the frontal aslant tract in speech planning: a neurosurgical case study. J. Cogn. Neurosci. 30, 752-769. doi: 10.1162/jocn_a_ 01244

Chicoine, A. J., Proteau, L., and Lassonde, M. (2000). Absence of interhemispheric transfer of unilateral visuomotor learning in young children and individuals with agenesis of the CC. Dev. Neuropsychol. 18, 73-94. doi: 10.1207/ S15326942DN1801_5

Choi, B. H. (1981). Radial glia of developing human fetal spinal cord: golgi, immunohistochemical and electron microscopic study. Brain Res. 227, 249267. doi: 10.1016/0165-3806(81)90112-7

Chung, S., Fieremans, E., Kucukboyaci, N. E., Wang, X., Morton, C. J., Novikov, D. S., et al. (2018). Working memory and brain tissue microstructure: WM tract integrity based on multi-shell diffusion MRI. Sci. Rep. 8:3175. doi: 10.1038/ s41598-018-21428-4

Collins, S. E., Spencer-Smith, M., Mürner-Lavanchy, I., Kelly, C. E., Pyman, P., Pascoe, L., et al. (2019). WM microstructure correlates with mathematics but not word reading performance in 13-year-old children born very preterm and full-term. NeuroImage Clin. 24:101944. doi: 10.1016/j.nicl.2019.101944

Conner, A. K., Briggs, R. G., Sali, G., Rahimi, M., Baker, C. M., Burks, J. D., et al. (2018). A connectomic atlas of the human cerebrum-chapter 13: tractographic description of the inferior frontooccipital fasciculus. Oper. Neurosurg. (Hagerstown) 15, S436-S443. doi: 10.1093/ons/opy267

Costa, A. R., Pinto-Costa, R., Sousa, S. C., and Sousa, M. M. (2018). The regulation of axon diameter: from axonal circumferential contractility to activity-dependent axon swelling. Front. Mol. Neurosci. 11:319. doi: 10.3389/ fnmol.2018.00319

De Bellis, M. D., Keshavan, M. S., Beers, S. R., Hall, J., Frustaci, K., Masalehdan, A., et al. (2001). Sex differences in brain maturation during childhood and adolescence. Cereb. Cortex 11, 552-557. doi: $10.1093 /$ cercor/11.6.552

De Faria, O., Gonsalvez, D. G., Nicholson, M., and Xiao, J. (2019). Activitydependent central nervous system myelination throughout life. J. Neurochem. 148, 447-461. doi: 10.1111/jnc. 14592

De Witt Hamer, P. C., Moritz-Gasser, S., Gatignol, P., and Duffau, H. (2011). Is the human left middle longitudinal fascicle essential for language? A brain electrostimulation study. Hum. Brain Mapp. 32, 962-973. doi: 10.1002/hbm. 21082

Dean, D. C., O’Muircheartaigh, J., Dirks, H., Travers, B. G., Adluru, N., Alexander, A. L., et al. (2016). Mapping an index of the myelin g-ratio in infants using magnetic resonance imaging. Neuroimage 132, 225-237. doi: 10.1016/j. neuroimage.2016.02.040

Dean, D. C., O’Muircheartaigh, J., Dirks, H., Waskiewicz, N., Lehman, K., Walker, L., et al. (2015). Estimating the age of healthy infants from quantitative myelin water fraction maps. Hum. Brain Mapp. 36, 1233-1244. doi: 10.1002/hbm. 22671

Dekaban, A. S. (1978). Changes in brain weights during the span of human life: relation of brain weights to body heights and body weights. Ann. Neurol. 4, 345-356. doi: 10.1002/ana.410040410

den Braber, A., Van't Ent, D., Stoffers, D., Linkenkaer-Hansen, K., Boomsma, D. I., and de Geus, E. J. C. (2013). Sex differences in gray and WM structure in agematched unrelated males and females and opposite-sex siblings. Int. J. Psychol. Res. 6, 7-21. doi: 10.21500/20112084.712

Deoni, S. C. L., Dean, D. C., O'Muircheartaigh, J., Dirks, H., and Jerskey, B. A. (2012). Investigating white matter development in infancy and early childhood using myelin water faction and relaxation time mapping. Neuroimage 63, 1038-1053. doi: 10.1016/j.neuroimage.2012.07.037

Deoni, S. C. L., Matthews, L., and Kolind, S. H. (2013). One component? Two components? Three? The effect of including a nonexchanging "free" water component in multicomponent driven equilibrium single pulse observation of $\mathrm{T} 1$ and T2. Magn. Reson. Med. 70, 147-154. doi: $10.1002 / \mathrm{mrm} .24429$
Deoni, S. C. L., Mercure, E., Blasi, A., Gasston, D., Thomson, A., Johnson, M., et al. (2011). Mapping infant brain myelination with magnetic resonance imaging. J. Neurosci. 31, 784-791. doi: 10.1523/JNEUROSCI.2106-10.2011

Derome, A. E. (ed.) (1987). "5 - The nuclear overhauser effect," in Tetrahedron Organic Chemistry Series Modern NMR Techniques for Chemistry Research, ed. A. E. Derome (Amsterdam: Elsevier), 97-127. doi: 10.1016/B978-0-08-0325132.50012-7

Desmond, D. W. (2002). Cognition and WM lesions. Cerebrovasc. Dis. 13 Suppl 2, 53-57. doi: 10.1159/000049151

Dick, A., Bernal, B., and Tremblay, P. (2013). The language connectome: new pathways, new concepts. Neurosci.Rev. J. Bringing Neurobiol. Neurol. Psychiatry 20, 453-467. doi: 10.1177/1073858413513502

Dick, A. S., Garic, D., Graziano, P., and Tremblay, P. (2019). The frontal aslant tract (FAT) and its role in speech, language and executive function. Cortex 111, 148-163. doi: 10.1016/j.cortex.2018.10.015

Dick, A. S., and Tremblay, P. (2012). Beyond the arcuate fasciculus: consensus and controversy in the connectional anatomy of language. Brain 135, 3529-3550. doi: 10.1093/brain/aws222

Doran, S. J., Jakob, P., and Décorps, M. (1996). Rapid repetition of the "burst" sequence: the role of diffusion and consequences for imaging. Magnetic Resonance in Medicine 35, 547-553. doi: 10.1002/mrm.1910350414

Du, Y. P., Chu, R., Hwang, D., Brown, M. S., Kleinschmidt-DeMasters, B. K., Singel, D., et al. (2007). Fast multislice mapping of the myelin water fraction using multicompartment analysis of T decay at 3T: a preliminary postmortem study. Magn. Reson. Med. 58, 865-870. doi: 10.1002/mrm.21409

Ducharme, J. R., Forest, M. G., De Peretti, E., Sempé, M., Collu, R., and Bertrand, J. (1976). Plasma adrenal and gonadal sex steroids in human pubertal development. J. Clin. Endocrinol. Metab. 42, 468-476. doi: 10.1210/jcem-42-3468

Duffau, H. (2019). "Awake craniotomies for neoplasms involving language networks," in Comprehensive Overview of Modern Surgical Approaches to Intrinsic Brain Tumors, Chap. 19, eds K. Chaichana and A. Quiñones-Hinojosa (Cambridge, MA: Academic Press), 329-345. doi: 10.1016/B978-0-12-8117835.00019-7

Duval, T., Lévy, S., Stikov, N., Campbell, J., Mezer, A., Witzel, T., et al. (2017). g-Ratio weighted imaging of the human spinal cord in vivo. Neuroimage 145, 11-23. doi: 10.1016/j.neuroimage.2016.09.018

Edzes, H. T., and Samulski, E. T. (1978). The measurement of cross-relaxation effects in the proton NMR spin-lattice relaxation of water in biological systems: hydrated collagen and muscle. J. Magn. Reson. (1969) 31, 207-229. doi: 10.1016/ 0022-2364(78)90185-3

Eliot, L., Ahmed, A., Khan, H., and Patel, J. (2021). Dump the "dimorphism": comprehensive synthesis of human brain studies reveals few male-female differences beyond size. Neurosci. Biobehav. Rev. 125, 667-697. doi: 10.1016/ j.neubiorev.2021.02.026

Ellerbrock, I., and Mohammadi, S. (2017). Four in vivo g-ratio-weighted imaging methods: comparability and repeatability at the group level. Hum. Brain Mapp. 39, 24-41. doi: 10.1002/hbm.23858

Engelbrecht, V., Rassek, M., Preiss, S., Wald, C., and Mödder, U. (1998). Agedependent changes in magnetization transfer contrast of WM in the pediatric brain. AJNR. Am. J. Neuroradiol. 19, 1923-1929.

Faizy, T. D., Thaler, C., Broocks, G., Flottmann, F., Leischner, H., Kniep, H., et al. (2020). The myelin water fraction serves as a marker for age-related myelin alterations in the cerebral white matter - a multiparametric MRI aging study. Front. Neurosci. 14:136. doi: 10.3389/fnins.2020.00136

Fields, R. D. (2010). Change in the brain's WM. Science 330, 768-769. doi: 10.1126/ science.1199139

Fields, R. D. (2015). A new mechanism of nervous system plasticity: activitydependent myelination. Nat. Rev. Neurosci. 16, 756-767. doi: 10.1038/nrn4023

Filley, C. M., and Fields, R. D. (2016). WM and cognition: making the connection. J. Neurophysiol. 116, 2093-2104. doi: 10.1152/jn.00221.2016

Fink, F., Eling, P., Rischkau, E., Beyer, N., Tomandl, B., Klein, J., et al. (2010). The association between California Verbal Learning Test performance and fibre impairment in multiple sclerosis: evidence from diffusion tensor imaging. Mult. Scler. 16, 332-341. doi: 10.1177/1352458509356367 
Fitsiori, A., Nguyen, D., Karentzos, A., Delavelle, J., and Vargas, M. I. (2011). The CC: WM or terra incognita. Br. J. Radiol. 84, 5-18. doi: 10.1259/bjr/21946513

Flechsig, P. (1901). Developmental (myelogenetic) localisation of the cerebral cortex in the human subject. Lancet 158, 1027-1030. doi: 10.1016/S01406736(01)01429-5

Flechsig, P. (1920). Anatomie des Menschlichen Gehirns und Rückenmarks auf Myelogenetischer Grundlage. Leipzig: G. Thieme.

Fleischman, D. A., Yang, J., Arfanakis, K., Arvanitakis, Z., Leurgans, S. E., Turner, A. D., et al. (2015). Physical activity, motor function, and WM hyperintensity burden in healthy older adults. Neurology 84, 1294-1300. doi: 10.1212/WNL. 0000000000001417

Flynn, S. W., Lang, D. J., Mackay, A. L., Goghari, V., Vavasour, I. M., Whittall, K. P., et al. (2003). Abnormalities of myelination in schizophrenia detected in vivo with MRI, and post-mortem with analysis of oligodendrocyte proteins. Mol. Psychiatry 8, 811-820. doi: 10.1038/sj.mp.4001337

Forkel, S., Friedrich, P., Thiebaut de Schotten, M., and Howells, H. (2020). WM variability, cognition, and disorders: a systematic review. medRxiv [Preprint] doi: 10.1101/2020.04.22.20075127

Frye, R. E., Hasan, K., Malmberg, B., Desouza, L., Swank, P., Smith, K., et al. (2010). Superior longitudinal fasciculus and cognitive dysfunction in adolescents born preterm and at term. Dev. Med. Child Neurol. 52, 760-766. doi: 10.1111/j.14698749.2010.03633.x

Fujie, S., Namiki, C., Nishi, H., Yamada, M., Miyata, J., Sakata, D., et al. (2008). The role of the uncinate fasciculus in memory and emotional recognition in amnestic mild cognitive impairment. Dement Geriatr. Cogn. Disord. 26, 432-439. doi: 10.1159/000165381

Fujii, M., Maesawa, S., Motomura, K., Futamura, M., Hayashi, Y., Koba, I., et al. (2015). Intraoperative subcortical mapping of a language-associated deep frontal tract connecting the superior frontal gyrus to Broca's area in the dominant hemisphere of patients with glioma. J. Neurosurg. 122, 1390-1396. doi: 10.3171/2014.10.JNS14945

Fung, B. M. (1986). Nuclear magnetic resonance study of water interactions with proteins, biomolecules, membranes, and tissues. Meth. Enzymol. 127, 151-161. doi: 10.1016/0076-6879(86)27013-5

Ganzetti, M., Wenderoth, N., and Mantini, D. (2014). Whole brain myelin mapping using T1- and T2-weighted MR imaging data. Front. Hum. Neurosci. 8:671. doi: $10.3389 /$ fnhum.2014.00671

Geschwind, N. (1970). The organization of language and the brain. Science 170, 940-944. doi: 10.1126/science.170.3961.940

Giedd, J. N., Blumenthal, J., Jeffries, N. O., Castellanos, F. X., Liu, H., Zijdenbos, A., et al. (1999). Brain development during childhood and adolescence: a longitudinal MRI study. Nat. Neurosci. 2, 861-863. doi: $10.1038 / 13158$

Giedd, J. N., Raznahan, A., Alexander-Bloch, A., Schmitt, E., Gogtay, N., and Rapoport, J. L. (2015). Child psychiatry branch of the national institute of mental health longitudinal structural magnetic resonance imaging study of human brain development. Neuropsychopharmacology 40, 43-49. doi: 10.1038/ npp.2014.236

Giedd, J. N., Rumsey, J. M., Castellanos, F. X., Rajapakse, J. C., Kaysen, D., Vaituzis, A. C., et al. (1996). A quantitative MRI study of the Corpus Callosum in children and adolescents. Brain Res. Dev. Brain Res. 91, 274-280. doi: 10.1016/01653806(95)00193-x

Gil-Robles, S., Carvallo, A., Jimenez, M. D. M., Gomez Caicoya, A., Martinez, R., Ruiz-Ocaña, C., et al. (2013). Double dissociation between visual recognition and picture naming: a study of the visual language connectivity using tractography and brain stimulation. Neurosurgery 72, 678-686. doi: 10.1227/ NEU.0b013e318282a361

Glasser, M. F., Goyal, M. S., Preuss, T. M., Raichle, M. E., and Van Essen, D. C. (2014). Trends and properties of human cerebral cortex: correlations with cortical myelin content. NeuroImage 93, 165-175. doi: 10.1016/j.neuroimage. 2013.03.060

Glasser, M. F., Sotiropoulos, S. N., Wilson, J. A., Coalson, T. S., Fischl, B., Andersson, J. L., et al. (2013). The minimal preprocessing pipelines for the Human Connectome Project. NeuroImage 80, 105-124. doi: 10.1016/j. neuroimage.2013.04.127

Glasser, M. F., and Van Essen, D. C. (2011). Mapping human cortical areas in vivo based on myelin content as revealed by T1- and T2-Weighted MRI. J. Neurosci. 31, 11597-11616. doi: 10.1523/JNEUROSCI.2180-11.2011
Groeschel, S., Vollmer, B., King, M. D., and Connelly, A. (2010). Developmental changes in cerebral grey and white matter volume from infancy to adulthood. Int. J. Dev. Neurosci. 28, 481-489. doi: 10.1016/j.ijdevneu.2010.06.004

Gupta, D. (2017). "Neuroanatomy," in Essentials of Neuroanesthesia, Chap. 1, ed. H. Prabhakar (Cambridge, MA: Academic Press), 3-40. doi: 10.1016/B978-012-805299-0.00001-4

Gur, R. C., Gunning-Dixon, F., Bilker, W. B., and Gur, R. E. (2002). Sex differences in temporo-limbic and frontal brain volumes of healthy adults. Cereb. Cortex 12, 998-1003. doi: 10.1093/cercor/12.9.998

Gur, R. C., Turetsky, B. I., Matsui, M., Yan, M., Bilker, W., Hughett, P., et al. (1999). Sex differences in brain gray and WM in healthy young adults: correlations with cognitive performance. J. Neurosci. 19, 4065-4072. doi: 10.1523/JNEUROSCI. 19-10-04065.1999

Han, Z., Ma, Y., Gong, G., He, Y., Caramazza, A., and Bi, Y. (2013). WM structural connectivity underlying semantic processing: evidence from brain damaged patients. Brain 136, 2952-2965. doi: 10.1093/brain/awt205

Harms, R. L., Fritz, F. J., Tobisch, A., Goebel, R., and Roebroeck, A. (2017). Robust and fast nonlinear optimization of diffusion MRI microstructure models. Neuroimage 155, 82-96. doi: 10.1016/j.neuroimage.2017.04.064

Hedman, A. M., van Haren, N. E. M., Schnack, H. G., Kahn, R. S., and Hulshoff Pol, H. E. (2012). Human brain changes across the life span: a review of 56 longitudinal magnetic resonance imaging studies. Hum. Brain Mapp. 33, 1987-2002. doi: 10.1002/hbm.21334

Heilbronner, S. R., and Haber, S. N. (2014). Frontal cortical and subcortical projections provide a basis for segmenting the cingulum bundle: implications for neuroimaging and psychiatric disorders. J. Neurosci. 34, 10041-10054. doi: 10.1523/JNEUROSCI.5459-13.2014

Helms, G., Draganski, B., Frackowiak, R., Ashburner, J., and Weiskopf, N. (2009). Improved segmentation of deep brain grey matter structures using magnetization transfer (MT) parameter maps. Neuroimage 47, 194-198. doi: 10.1016/j.neuroimage.2009.03.053

Henkelman, R. M., Stanisz, G. J., and Graham, S. J. (2001). Magnetization transfer in MRI: a review. NMR Biomed. 14, 57-64. doi: 10.1002/nbm.683

Herbet, G., Moritz-Gasser, S., and Duffau, H. (2017). Direct evidence for the contributive role of the right inferior fronto-occipital fasciculus in non-verbal semantic cognition. Brain Struct. Funct. 222, 1597-1610. doi: 10.1007/s00429016-1294-x

Hickok, G., and Poeppel, D. (2007). The cortical organization of speech processing. Nat. Rev. Neurosci. 8, 393-402. doi: 10.1038/nrn2113

Hill, R. A., Li, A. M., and Grutzendler, J. (2018). Lifelong cortical myelin plasticity and age-related degeneration in the live mammalian brain. Nat. Neurosci. 21, 683-695. doi: 10.1038/s41593-018-0120-6

Hiyoshi-Taniguchi, K., Oishi, N., Namiki, C., Miyata, J., Murai, T., Cichocki, A., et al. (2015). The uncinate fasciculus as a predictor of conversion from amnestic mild cognitive impairment to Alzheimer Disease. J. Neuroimaging 25, 748-753. doi: $10.1111 /$ jon. 12196

Hoeft, F., Barnea-Goraly, N., Haas, B. W., Golarai, G., Ng, D., Mills, D., et al. (2007). More is not always better: increased fractional anisotropy of superior longitudinal fasciculus associated with poor visuospatial abilities in Williams syndrome. J. Neurosci. 27, 11960-11965. doi: 10.1523/JNEUROSCI.3591-07. 2007

Hollund, I. M. H., Olsen, A., Skranes, J., Brubakk, A.-M., Håberg, A. K., Eikenes, L., et al. (2017). WM alterations and their associations with motor function in young adults born preterm with very low birth weight. Neuroimage Clin. 17, 241-250. doi: 10.1016/j.nicl.2017.10.006

Hughes, E. G., Kang, S. H., Fukaya, M., and Bergles, D. E. (2013). Oligodendrocyte progenitors balance growth with self-repulsion to achieve homeostasis in the adult brain. Nat. Neurosci. 16, 668-676. doi: 10.1038/nn.3390

Hughes, E. G., Orthmann-Murphy, J. L., Langseth, A. J., and Bergles, D. E. (2018). Myelin remodeling through experience-dependent oligodendrogenesis in the adult somatosensory cortex. Nat. Neurosci. 21, 696-706. doi: 10.1038/s41593018-0121-5

Hwang, S. J., Ji, E.-K., Lee, E.-K., Kim, Y. M., Shin, D. Y., Cheon, Y. H., et al. (2004). Gender differences in the corpus callosum of neonates. Neuroreport 15, 1029-1032. doi: 10.1097/00001756-200404290-00019

Indefrey, P., and Levelt, W. J. M. (2004). The spatial and temporal signatures of word production components. Cognition 92, 101-144. doi: 10.1016/j.cognition. 2002.06.001 
Itoyama, Y., Sternberger, N. H., Webster, H. D., Quarles, R. H., Cohen, S. R., and Richardson, E. P. (1980). Immunocytochemical observations on the distribution of myelin-associated glycoprotein and myelin basic protein in multiple sclerosis lesions. Ann. Neurol. 7, 167-177. doi: 10.1002/ana.410070212

Jäncke, L. (2018). Sex/gender differences in cognition, neurophysiology, and neuroanatomy. F1000Research 7, F1000FacultyRev-805. doi: 10.12688/f1000research.13917.1

Johansen-Berg, H., and Behrens, T. E. J. (2009). Diffusion MRI: From Quantitative Measurement to In-Vivo Neuroanatomy. Cambridge, MA: Academic Press.

Johansen-Berg, H., Della-Maggiore, V., Behrens, T. E. J., Smith, S. M., and Paus, T. (2007). Integrity of WM in the Corpus Callosum correlates with bimanual coordination skills. NeuroImage 36, T16-T21. doi: 10.1016/j.neuroimage.2007.03. 041

Jones, D. K., Christiansen, K. F., Chapman, R. J., and Aggleton, J. P. (2013a). Distinct subdivisions of the cingulum bundle revealed by diffusion MRI fibre tracking: Implications for neuropsychological investigations. Neuropsychologia 51, 67-78. doi: 10.1016/j.neuropsychologia.2012.11.018

Jones, D. K., Knösche, T. R., and Turner, R. (2013b). White matter integrity, fiber count, and other fallacies: the do's and don'ts of diffusion MRI. Neuroimage 73, 239-254. doi: 10.1016/j.neuroimage.2012.06.081

Kaes, T. (1907). Die Grosshirnrinde des Menschen in ihren Maßen und in ihrem Fasergehalt. Ein gehirnanatomischer Atlas (The Cerebral Cortex of Humans in Their Dimensions and Their Fiber Content. An Anatomical Brain Atlas). Jena: G. Fischer.

Kamali, A., Flanders, A. E., Brody, J., Hunter, J. V., and Hasan, K. M. (2014). Tracing superior longitudinal fasciculus connectivity in the human brain using high resolution diffusion tensor tractography. Brain Struct. Funct. 219, 269-281. doi: 10.1007/s00429-012-0498-y

Kanaan, R. A., Allin, M., Picchioni, M., Barker, G. J., Daly, E., Shergill, S. S., et al. (2012). Gender differences in WM microstructure. PLoS One 7:e38272. doi: 10.1371/journal.pone.0038272

Kantarci, K., Murray, M. E., Schwarz, C. G., Reid, R., Przybelski, S. A., Lesnick, T., et al. (2017). WM integrity on DTI and the pathologic staging of Alzheimer's disease. Neurobiol. Aging 56, 172-179. doi: 10.1016/j.neurobiolaging.2017.04. 024

Kantarci, K., Senjem, M. L., Avula, R., Zhang, B., Samikoglu, A. R., Weigand, S. D., et al. (2011). Diffusion tensor imaging and cognitive function in older adults with no dementia. Neurology 77, 26-34. doi: 10.1212/WNL.0b013e31822313dc

Kemerdere, R., de Champfleur, N. M., Deverdun, J., Cochereau, J., Moritz-Gasser, S., Herbet, G., et al. (2016). Role of the left frontal aslant tract in stuttering: a brain stimulation and tractographic study. J. Neurol. 263, 157-167. doi: 10. 1007/s00415-015-7949-3

Kochunov, P., Williamson, D. E., Lancaster, J., Fox, P., Cornell, J., Blangero, J., et al. (2012). Fractional anisotropy of water diffusion in cerebral white matter across the lifespan. Neurobiol. Aging 33, 9-20. doi: 10.1016/j.neurobiolaging.2010.01. 014

Koenig, S. H. (1991). Cholesterol of myelin is the determinant of gray-white contrast in MRI of brain. Magn. Reson. Med. 20, 285-291. doi: 10.1002/mrm. 1910200210

Krogsrud, S. K., Fjell, A. M., Tamnes, C. K., Grydeland, H., Mork, L., DueTønnessen, P., et al. (2016). Changes in WM microstructure in the developing brain - A longitudinal diffusion tensor imaging study of children from 4 to 11 years of age. Neuroimage 124, 473-486. doi: 10.1016/j.neuroimage.2015.09.017

Kucharczyk, W., Macdonald, P. M., Stanisz, G. J., and Henkelman, R. M. (1994). Relaxivity and magnetization transfer of white matter lipids at MR imaging: importance of cerebrosides and pH. Radiology 192, 521-529. doi: 10.1148/ radiology.192.2.8029426

Kulikova, S. P. (2015). Integration of Multimodal Imaging Data for Investigation of Brain Development. Doctorat de Neurosciences. thesis, Université Sorbonne, Paris.

Kumar, R., Chavez, A. S., Macey, P. M., Woo, M. A., and Harper, R. M. (2013). Brain axial and radial diffusivity changes with age and gender in healthy adults. Brain Res. 1512, 22-36. doi: 10.1016/j.brainres.2013.03.028

La Corte, E., Eldahaby, D., Greco, E., Aquino, D., Bertolini, G., Levi, V., et al. (2021). The frontal aslant tract: a systematic review for neurosurgical applications. Front. Neurol. 12:641586. doi: 10.3389/fneur.2021.641586

Labadie, C., Lee, J.-H., Rooney, W. D., Jarchow, S., Aubert-Frécon, M., Springer, C. S., et al. (2014). Myelin water mapping by spatially regularized longitudinal relaxographic imaging at high magnetic fields. Magn. Reson. Med. 71, 375-387. doi: $10.1002 / \mathrm{mrm} .24670$

Lancaster, J. L., Andrews, T., Hardies, L. J., Dodd, S., and Fox, P. T. (2003). Threepool model of white matter. J. Magn. Reson. Imaging 17, 1-10. doi: 10.1002/ jmri.10230

Lawes, I. N. C., Barrick, T. R., Murugam, V., Spierings, N., Evans, D. R., Song, M., et al. (2008). Atlas-based segmentation of white matter tracts of the human brain using diffusion tensor tractography and comparison with classical dissection. Neuroimage 39, 62-79. doi: 10.1016/j.neuroimage.2007.06.041

Lebel, C., and Beaulieu, C. (2009). Lateralization of the arcuate fasciculus from childhood to adulthood and its relation to cognitive abilities in children. Hum. Brain Mapp. 30, 3563-3573. doi: 10.1002/hbm.20779

Lebel, C., and Beaulieu, C. (2011). Longitudinal development of human brain wiring continues from childhood into adulthood. J. Neurosci. 31, 10937-10947. doi: 10.1523/JNEUROSCI.5302-10.2011

Lebel, C., and Deoni, S. (2018). The development of brain WM microstructure. Neuroimage 182, 207-218. doi: 10.1016/j.neuroimage.2017.12.097

Lebel, C., Gee, M., Camicioli, R., Wieler, M., Martin, W., and Beaulieu, C. (2012). Diffusion tensor imaging of WM tract evolution over the lifespan. Neuroimage 60, 340-352. doi: 10.1016/j.neuroimage.2011.11.094

Lebel, C., Shaywitz, B., Holahan, J., Shaywitz, S., Marchione, K., and Beaulieu, C. (2013). Diffusion tensor imaging correlates of reading ability in dysfluent and non-impaired readers. Brain Lang. 125, 215-222. doi: 10.1016/j.bandl.2012.10.009

Lebel, C., Treit, S., and Beaulieu, C. (2019). A review of diffusion MRI of typical WM development from early childhood to young adulthood. NMR Biomed. 32:e3778. doi: 10.1002/nbm.3778

Lebel, C., Walker, L., Leemans, A., Phillips, L., and Beaulieu, C. (2008). Microstructural maturation of the human brain from childhood to adulthood. Neuroimage 40, 1044-1055. doi: 10.1016/j.neuroimage.2007.12.053

Leng, Y., Shi, Y., Yu, Q., Van Horn, J. D., Tang, H., Li, J., et al. (2016). Phenotypic and genetic correlations between the lobar segments of the inferior frontooccipital fasciculus and attention. Sci. Rep. 6:33015. doi: 10.1038/srep33015

Leuze, C. W. U., Anwander, A., Bazin, P.-L., Dhital, B., Stüber, C., Reimann, K., et al. (2014). Layer-specific intracortical connectivity revealed with diffusion MRI. Cereb. Cortex 24, 328-339. doi: 10.1093/cercor/bhs311

Lin, Y.-C., Shih, Y.-C., Tseng, W.-Y. I., Chu, Y.-H., Wu, M.-T., Chen, T.-F., et al. (2014). Cingulum correlates of cognitive functions in patients with mild cognitive impairment and early Alzheimer's disease: a diffusion spectrum imaging study. Brain Topogr. 27, 393-402. doi: 10.1007/s10548-013-0346-2

Lindberg, P. G., Feydy, A., and Maier, M. A. (2010). White matter organization in cervical spinal cord relates differently to age and control of grip force in healthy subjects. J. Neurosci. 30, 4102-4109. doi: 10.1523/JNEUROSCI.5529-09.2010

Luders, E., Narr, K. L., Bilder, R. M., Thompson, P. M., Szeszko, P. R., Hamilton, L., et al. (2007). Positive correlations between corpus callosum thickness and intelligence. Neuroimage 37, 1457-1464. doi: 10.1016/j.neuroimage.2007.06.028

Ludwig, E., and Klingler, J. (1956). Atlas Cerebri Humani-The Inner Structure of the Brain. Basel: Karger.

Lynn, J., Anand, C., Arshad, M., Khatib, D., Stanley, J., and Raz, N. (2019). Lifespan age-related differences in the regional white matter microstructure of the human corpus callosum. Innov. Aging 3:S945. doi: 10.1093/geroni/igz038.3435

Mabbott, D. J., Rovet, J., Noseworthy, M. D., Smith, M. L., and Rockel, C. (2009). The relations between WM and declarative memory in older children and adolescents. Brain Res. 1294, 80-90. doi: 10.1016/j.brainres.2009.07.046

MacKay, A., Whittall, K., Adler, J., Li, D., Paty, D., and Graeb, D. (1994). In vivo visualization of myelin water in brain by magnetic resonance. Magn. Reson. Med. 31, 673-677. doi: 10.1002/mrm.1910310614

MacKay, A. L., and Laule, C. (2016). Magnetic resonance of myelin water: an in vivo marker for myelin. Brain Plast 2, 71-91. doi: 10.3233/BPL-160033

Makris, N., Kennedy, D. N., McInerney, S., Sorensen, A. G., Wang, R., Caviness, V. S., et al. (2005). Segmentation of subcomponents within the superior longitudinal fascicle in humans: a quantitative, in vivo, DT-MRI study. Cereb. Cortex 15, 854-869. doi: 10.1093/cercor/bhh186

Makris, N., Papadimitriou, G. M., Kaiser, J. R., Sorg, S., Kennedy, D. N., and Pandya, D. N. (2009). Delineation of the middle longitudinal fascicle in humans: a quantitative, in vivo, DT-MRI study. Cereb. Cortex 19, 777-785. doi: 10.1093/ cercor/bhn 124 
Mandonnet, E., Nouet, A., Gatignol, P., Capelle, L., and Duffau, H. (2007). Does the left inferior longitudinal fasciculus play a role in language? A brain stimulation study. Brain 130, 623-629. doi: 10.1093/brain/awl361

Mandonnet, E., Sarubbo, S., and Petit, L. (2018). The nomenclature of human WM association pathways: proposal for a systematic taxonomic anatomical classification. Front. Neuroanat. 12:94. doi: 10.3389/fnana.2018.00094

Martino, J., Brogna, C., Robles, S. G., Vergani, F., and Duffau, H. (2010). Anatomic dissection of the inferior fronto-occipital fasciculus revisited in the lights of brain stimulation data. Cortex 46, 691-699. doi: 10.1016/j.cortex.2009.07.015

McDonald, C. R., Ahmadi, M. E., Hagler, D. J., Tecoma, E. S., Iragui, V. J., Gharapetian, L., et al. (2008). Diffusion tensor imaging correlates of memory and language impairments in temporal lobe epilepsy. Neurology 71, 1869-1876. doi: 10.1212/01.wnl.0000327824.05348.3b

McKenzie, I. A., Ohayon, D., Li, H., de Faria, J. P., Emery, B., Tohyama, K., et al. (2014). Motor skill learning requires active central myelination. Science 346, 318-322. doi: 10.1126/science. 1254960

Mehta, R. C., Pike, G. B., and Enzmann, D. R. (1995). Magnetization transfer MR of the normal adult brain. AJNR Am. J. Neuroradiol. 16, 2085-2091.

Menjot de Champfleur, N., Lima Maldonado, I., Moritz-Gasser, S., Machi, P., Le Bars, E., Bonafé, A., et al. (2013). Middle longitudinal fasciculus delineation within language pathways: a diffusion tensor imaging study in human. Eur. J. Radiol. 82, 151-157. doi: 10.1016/j.ejrad.2012.05.034

Mezer, A., Yeatman, J. D., Stikov, N., Kay, K. N., Cho, N.-J., Dougherty, R. F., et al. (2013). Quantifying the local tissue volume and composition in individual brains with magnetic resonance imaging. Nat. Med. 19, 1667-1672. doi: 10 . 1038/nm.3390

Micheva, K. D., Wolman, D., Mensh, B. D., Pax, E., Buchanan, J., Smith, S. J., et al. (2016). A large fraction of neocortical myelin ensheathes axons of local inhibitory neurons. eLife 5:e15784. doi: 10.7554/eLife.15784

Miot-Noirault, E., Barantin, L., Akoka, S., and Le Pape, A. (1997). T2 relaxation time as a marker of brain myelination: experimental MR study in two neonatal animal models. J. Neurosci. Methods 72, 5-14. doi: 10.1016/S0165-0270(96) 00148-3

Mishra, A., Wang, F., Chen, L. M., and Gore, J. C. (2020). Longitudinal changes in DTI parameters of specific spinal white matter tracts correlate with behavior following spinal cord injury in monkeys. Sci. Rep. 10:17316. doi: 10.1038/ s41598-020-74234-2

Mori, S., and Zhang, J. (2006). Principles of diffusion tensor imaging and its applications to basic neuroscience research. Neuron 51, 527-539. doi: 10.1016/ j.neuron.2006.08.012

Moritz-Gasser, S., Herbet, G., and Duffau, H. (2013). Mapping the connectivity underlying multimodal (verbal and non-verbal) semantic processing: a brain electrostimulation study. Neuropsychologia 51, 1814-1822. doi: 10.1016/j. neuropsychologia.2013.06.007

Morris, S. R., Holmes, R. D., Dvorak, A. V., Liu, H., Yoo, Y., Vavasour, I. M., et al. (2020). Brain myelin water fraction and diffusion tensor imaging atlases for 9-10 year-old children. J. Neuroimaging 30, 150-160. doi: 10.1111/jon.12689

Nave, K.-A., and Werner, H. B. (2014). Myelination of the nervous system: mechanisms and functions. Annu. Rev. Cell Dev. Biol. 30, 503-533. doi: 10.1146/ annurev-cellbio-100913-013101

Niogi, S. N., Mukherjee, P., Ghajar, J., Johnson, C. E., Kolster, R., Lee, H., et al. (2008). Structural dissociation of attentional control and memory in adults with and without mild traumatic brain injury. Brain 131, 3209-3221. doi: 10.1093/ brain/awn 247

O'Donnell, L. J., and Westin, C.-F. (2011). An introduction to diffusion tensor image analysis. Neurosurg. Clin. N Am. 22, 185-196,viii. doi: 10.1016/j.nec.2010. 12.004

Oh, J., Han, E. T., Pelletier, D., and Nelson, S. J. (2006). Measurement of in vivo multi-component T2 relaxation times for brain tissue using multi-slice T2 prep at 1.5 and 3 T. Magn. Reson. Imaging 24, 33-43. doi: 10.1016/j.mri.2005.10.016

Ohlhauser, L., Parker, A. F., Smart, C. M., and Gawryluk, J. R. (2018). WM and its relationship with cognition in subjective cognitive decline. Alzheimers Dement (Amst) 11, 28-35. doi: 10.1016/j.dadm.2018.10.008

Olson, I. R., Heide, R. J. V. D., Alm, K. H., and Vyas, G. (2015). Development of the uncinate fasciculus: Implications for theory and developmental disorders. Dev. Cogn. Neurosci. 14, 50-61. doi: 10.1016/j.dcn.2015.06.003

Ortibus, E., Verhoeven, J., Sunaert, S., Casteels, I., de Cock, P., and Lagae, L. (2012). Integrity of the inferior longitudinal fasciculus and impaired object recognition in children: a diffusion tensor imaging study. Dev. Med. Child Neurol. 54, 38-43. doi: 10.1111/j.1469-8749.2011.04147.x

Pajevic, S., and Pierpaoli, C. (1999). Color schemes to represent the orientation of anisotropic tissues from diffusion tensor data: application to WM fiber tract mapping in the human brain. Magn. Reson. Med. 42, 526-540. doi: 10.1002/ (sici) 1522-2594(199909)42:3<526::aid-mrm15>3.0.co;2-j

Panesar, S. S., Yeh, F.-C., Jacquesson, T., Hula, W., and Fernandez-Miranda, J. C. (2018). A quantitative tractography study into the connectivity, segmentation and laterality of the human inferior longitudinal fasciculus. Front. Neuroanat. 12:47. doi: 10.3389/fnana.2018.00047

Papagno, C., Miracapillo, C., Casarotti, A., Romero Lauro, L. J., Castellano, A., Falini, A., et al. (2011). What is the role of the uncinate fasciculus? Surgical removal and proper name retrieval. Brain 134, 405-414. doi: 10.1093/brain/ awq283

Papez, J. W. (1995). A proposed mechanism of emotion. 1937. J. Neuropsychiatry Clin. Neurosci. 7, 103-112. doi: 10.1176/jnp.7.1.103

Pascual-Leone, J. (1970). A mathematical model for the transition rule in Piaget's developmental stages. Acta Psychol. Amsterdam 32, 301-345. doi: 10.1016/ 0001-6918(70)90108-3

Pascual-Leone, J., and Johnson, J. (2021). The Working Mind: Meaning and Mental Attention in Human Development. Cambridge, MA: MIT Press.

Passe, T. J., Rajagopalan, P., Tupler, L. A., Byrum, C. E., MacFall, J. R., and Krishnan, K. R. (1997). Age and sex effects on brain morphology. Prog. Neuropsychopharmacol. Biol. Psychiatry 21, 1231-1237. doi: 10.1016/s02785846(97)00160-7

Patel, R., Moore, S., Crawford, D. K., Hannsun, G., Sasidhar, M. V., Tan, K., et al. (2013). Attenuation of corpus callosum axon myelination and remyelination in the absence of circulating sex hormones. Brain Pathol. 23, 462-475. doi: 10.1111/bpa.12029

Paul, L. K., Corsello, C., Kennedy, D. P., and Adolphs, R. (2014). Agenesis of the Corpus Callosum and autism: a comprehensive comparison. Brain 137, 1813-1829. doi: 10.1093/brain/awu070

Paul, L. K., Van Lancker-Sidtis, D., Schieffer, B., Dietrich, R., and Brown, W. S. (2003). Communicative deficits in agenesis of the CC: nonliteral language and affective prosody. Brain Lang. 85, 313-324. doi: 10.1016/s0093-934x(03)000 $62-2$

Paus, T. (2010). Growth of white matter in the adolescent brain: myelin or axon? Brain Cogn. 72, 26-35. doi: 10.1016/j.bandc.2009.06.002

Paus, T., Collins, D. L., Evans, A. C., Leonard, G., Pike, B., and Zijdenbos, A. (2001). Maturation of WM in the human brain: a review of magnetic resonance studies. Brain Res. Bull. 54, 255-266. doi: 10.1016/s0361-9230(00)00434-2

Peters, B. D., Szeszko, P. R., Radua, J., Ikuta, T., Gruner, P., DeRosse, P., et al. (2012). WM development in adolescence: diffusion tensor imaging and metaanalytic results. Schizophr. Bull. 38, 1308-1317. doi: 10.1093/schbul/sbs054

Petrides, M., and Pandya, D. N. (2002). "Association pathways of the prefrontal cortex and functional observations," in Principles of Frontal Lobe Function, eds D. T. Stuss and R. T. Knight ((New York, NY: Oxford University Press), 31-50. doi: 10.1093/acprof:oso/9780195134971.003.0003

Pryweller, J. R., Schauder, K. B., Anderson, A. W., Heacock, J. L., FossFeig, J. H., Newsom, C. R., et al. (2014). WM correlates of sensory processing in autism spectrum disorders. Neuroimage Clin. 6, 379-387. doi: 10.1016/j.nicl.2014.09.018

Rademacher, J., Engelbrecht, V., Bürgel, U., Freund, H., and Zilles, K. (1999). Measuring in vivo myelination of human white matter fiber tracts with magnetization transfer MR. Neuroimage 9, 393-406. doi: 10.1006/nimg.1998. 0416

Reynolds, B. B., By, S., Weinberg, Q. R., Witt, A. A., Newton, A. T., Feiler, H. R., et al. (2019). Quantification of DTI in the pediatric spinal cord: application to clinical evaluation in a healthy patient population. AJNR Am. J. Neuroradiol. 40, 1236-1241. doi: 10.3174/ajnr.A6104

Ritchie, S. J., Cox, S. R., Shen, X., Lombardo, M. V., Reus, L. M., Alloza, C., et al. (2018). Sex differences in the adult human brain: evidence from 5216 UK biobank participants. Cereb. Cortex 28, 2959-2975. doi: 10.1093/cercor/ bhy 109

Rivers, L. E., Young, K. M., Rizzi, M., Jamen, F., Psachoulia, K., Wade, A., et al. (2008). PDGFRA/NG2 glia generate myelinating oligodendrocytes and piriform projection neurons in adult mice. Nat. Neurosci. 11, 1392-1401. doi: $10.1038 / \mathrm{nn} .2220$ 
Rizio, A. A., and Diaz, M. T. (2016). Language, aging, and cognition: Frontal aslant tract and superior longitudinal fasciculus contribute to working memory performance in older adults. Neuroreport 27, 689-693. doi: 10.1097/WNR. 0000000000000597

Rofes, A., Talacchi, A., Santini, B., Zocatelli, G., Alessandrini, F., Nickels, L., et al. (2014). "The language-role of the inferior fronto-occipital fasciculus: Evidence from awake surgery," in Proceedings of the Rovereto Workshop on Concepts, Actions, and Objects: Functional and Neural Perspectives, (Trento: University of Trento).

Rollans, C., Cheema, K., Georgiou, G. K., and Cummine, J. (2017). Pathways of the inferior frontal occipital fasciculus in overt speech and reading. Neuroscience 364, 93-106. doi: 10.1016/j.neuroscience.2017.09.011

Rushton, W. a. H (1951). A theory of the effects of fibre size in medullated nerve. J. Physiol. 115, 101-122. doi: 10.1113/jphysiol.1951.sp004655

Saksena, S., Middleton, D. M., Krisa, L., Shah, P., Faro, S. H., Sinko, R., et al. (2016). Diffusion tensor imaging of the normal cervical and thoracic pediatric spinal cord. AJNR Am. J. Neuroradiol. 37, 2150-2157. doi: 10.3174/ajnr.A4883

Sampaio-Baptista, C., Khrapitchev, A. A., Foxley, S., Schlagheck, T., Scholz, J., Jbabdi, S., et al. (2013). Motor skill learning induces changes in WM microstructure and myelination. J. Neurosci. 33, 19499-19503. doi: 10.1523/ JNEUROSCI.3048-13.2013

Sato, T., Maruyama, N., Hoshida, T., and Minato, K. (2012). Correlation between uncinate fasciculus and memory tasks in healthy individual using diffusion tensor tractography. Conf. Proc. IEEE Eng. Med. Biol. Soc. 2012, 424-427. doi: 10.1109/EMBC.2012.6345958

Saur, D., Kreher, B. W., Schnell, S., Kümmerer, D., Kellmeyer, P., Vry, M.-S., et al. (2008). Ventral and dorsal pathways for language. Proc. Natl. Acad. Sci. U.S.A. 105, 18035-18040. doi: 10.1073/pnas.0805234105

Saur, D., Schelter, B., Schnell, S., Kratochvil, D., Küpper, H., Kellmeyer, P., et al. (2010). Combining functional and anatomical connectivity reveals brain networks for auditory language comprehension. Neuroimage 49, 3187-3197. doi: 10.1016/j.neuroimage.2009.11.009

Schmahmann, J., and Pandya, D. (2006). Fiber Pathways of the Brain. New York, NY: Oxford University Press, doi: 10.1093/acprof:oso/9780195104233.003.0014

Schmied, A., Soda, T., Gerig, G., Styner, M., Swanson, M. R., Elison, J. T., et al. (2020). Sex differences associated with Corpus Callosum development in human infants: a longitudinal multimodal imaging study. NeuroImage 215, 116821. doi: 10.1016/j.neuroimage.2020.116821

Schmithorst, V. J., Holland, S. K., and Dardzinski, B. J. (2008). Developmental differences in WM architecture between boys and girls. Hum. Brain Mapp. 29, 696-710. doi: 10.1002/hbm.20431

Schmithorst, V. J., Wilke, M., Dardzinski, B. J., and Holland, S. K. (2002). Correlation of WM diffusivity and anisotropy with age during childhood and adolescence: a cross-sectional diffusion-tensor MR imaging study. Radiology 222, 212-218. doi: 10.1148/radiol.2221010626

Scholz, J., Tomassini, V., and Johansen-Berg, H. (2009). "Individual differences in WM microstructure in the healthy brain," in Diffusion MRI, Chap. 11, eds H. Johansen-Berg and T. E. J. Behrens (San Diego, CA: Academic Press), 237-249. doi: 10.1016/B978-0-12-374709-9.00011-0

Sellers, K. J., Erli, F., Raval, P., Watson, I. A., Chen, D., and Srivastava, D. P. (2015). Rapid modulation of synaptogenesis and spinogenesis by $17 \beta$-estradiol in primary cortical neurons. Front. Cell Neurosci. 9:137. doi: 10.3389/fncel.2015. 00137

Sexton, C. E., Walhovd, K. B., Storsve, A. B., Tamnes, C. K., Westlye, L. T., Johansen-Berg, H., et al. (2014). Accelerated changes in WM microstructure during aging: a longitudinal diffusion tensor imaging study. J. Neurosci. 34, 15425-15436. doi: 10.1523/JNEUROSCI.0203-14.2014

Shin, J., Rowley, J., Chowdhury, R., Jolicoeur, P., Klein, D., Grova, C., et al. (2019). Inferior longitudinal fasciculus' role in visual processing and language comprehension: a combined MEG-DTI study. Front. Neurosci. 13:875. doi: 10.3389/fnins.2019.00875

Shin, Y.-W., Kim, D. J., Ha, T. H., Park, H.-J., Moon, W.-J., Chung, E. C., et al. (2005). Sex differences in the human CC: diffusion tensor imaging study. Neuroreport 16, 795-798. doi: 10.1097/00001756-200505310-00003

Shinoura, N., Suzuki, Y., Tsukada, M., Katsuki, S., Yamada, R., Tabei, Y., et al. (2007). Impairment of inferior longitudinal fasciculus plays a role in visual memory disturbance. Neurocase 13, 127-130. doi: 10.1080/13554790701399254
Singh, S., Singh, K., Trivedi, R., Goyal, S., Kaur, P., Singh, N., et al. (2016). Microstructural abnormalities of uncinate fasciculus as a function of impaired cognition in schizophrenia: a DTI study. J. Biosci. 41, 419-426. doi: 10.1007/ s12038-016-9631-z

Singhi, S., Tekes, A., Thurnher, M., Gilson, W. D., Izbudak, I., Thompson, C. B., et al. (2012). Diffusion tensor imaging of the maturing paediatric cervical spinal cord: from the neonate to the young adult. J. Neuroradiol. 39, 142-148. doi: 10.1016/j.neurad.2011.05.002

Sled, J. G., and Pike, G. B. (2001). Quantitative imaging of magnetization transfer exchange and relaxation properties in vivo using MRI. Magn. Reson. Med. 46, 923-931. doi: 10.1002/mrm.1278

Smith, C. C., Vedder, L. C., and McMahon, L. L. (2009). Estradiol and the relationship between dendritic spines, NR2B containing NMDA receptors, and the magnitude of long-term potentiation at hippocampal CA3-CA1 synapses. Psychoneuroendocrinology 34S1, S130-S142. doi: 10.1016/j.psyneuen.2009.06. 003

Snaidero, N., Möbius, W., Czopka, T., Hekking, L. H. P., Mathisen, C., Verkleij, D., et al. (2014). Myelin membrane wrapping of CNS Axons by PI(3,4,5)P3dependent polarized growth at the inner tongue. Cell 156, 277-290. doi: 10. 1016/j.cell.2013.11.044

Snaidero, N., and Simons, M. (2014). Myelination at a glance. J. Cell. Sci. 127, 2999-3004. doi: 10.1242/jcs.151043

Srivastava, D. P., and Penzes, P. (2011). Rapid estradiol modulation of neuronal connectivity and its implications for disease. Front. Endocrinol (Lausanne) 2:77. doi: 10.3389/fendo.2011.00077

Stedehouder, J., Brizee, D., Shpak, G., and Kushner, S. A. (2018). Activitydependent myelination of parvalbumin interneurons mediated by axonal morphological plasticity. J. Neurosci. 38, 3631-3642. doi: 10.1523/JNEUROSCI. 0074-18.2018

Stejskal, E. O., and Tanner, J. E. (1965). Spin diffusion measurements: spin echoes in the presence of a time-dependent field gradient. J. Chem. Phys. 42, 288-292. doi: $10.1063 / 1.1695690$

Stepanyants, A., Martinez, L. M., Ferecskó, A. S., and Kisvárday, Z. F. (2009). The fractions of short- and long-range connections in the visual cortex. Proc. Natl. Acad. Sci. U.S.A. 106, 3555-3560. doi: 10.1073/pnas.0810390106

Stocker, S., Güttinger, H. R., and Herth, G. (1994). Exogenous testosterone differentially affects myelination and neurone soma sizes in the brain of canaries. Neuroreport 5, 1449-1452. doi: 10.1097/00001756-199407000-00010

Stüber, C., Morawski, M., Schäfer, A., Labadie, C., Wähnert, M., Leuze, C., et al. (2014). Myelin and iron concentration in the human brain: a quantitative study of MRI contrast. Neuroimage 93(Pt 1), 95-106. doi: 10.1016/j.neuroimage.2014. 02.026

Sullivan, E. V., Rohlfing, T., and Pfefferbaum, A. (2010). Quantitative fiber tracking of lateral and interhemispheric WM systems in normal aging: relations to timed performance. Neurobiol. Aging 31, 464-481. doi: 10.1016/j.neurobiolaging. 2008.04.007

Szeszko, P. R., Vogel, J., Ashtari, M., Malhotra, A. K., Bates, J., Kane, J. M., et al. (2003). Sex differences in frontal lobe WM microstructure: a DTI study. Neuroreport 14, 2469-2473. doi: 10.1097/00001756-200312190-00035

Taddei, M., Tettamanti, M., Zanoni, A., Cappa, S., and Battaglia, M. (2012). Brain WM organisation in adolescence is related to childhood cerebral responses to facial expressions and harm avoidance. Neuroimage 61, 1394-1401. doi: 10.1016/j.neuroimage.2012.03.062

Takahashi, M., Iwamoto, K., Fukatsu, H., Naganawa, S., Iidaka, T., and Ozaki, N. (2010). WM microstructure of the cingulum and cerebellar peduncle is related to sustained attention and working memory: a diffusion tensor imaging study. Neurosci. Lett. 477, 72-76. doi: 10.1016/j.neulet.2010.04.031

Takeuchi, H., Sekiguchi, A., Taki, Y., Yokoyama, S., Yomogida, Y., Komuro, N., et al. (2010). Training of working memory impacts structural connectivity. J. Neurosci. 30, 3297-3303. doi: 10.1523/JNEUROSCI.4611-09. 2010

Tamnes, C. K., Roalf, D. R., Goddings, A.-L., and Lebel, C. (2018). Diffusion MRI of WM microstructure development in childhood and adolescence: Methods, challenges and progress. Devel. Cogn. Neurosci. 33, 161-175. doi: 10.1016/j.dcn. 2017.12.002

Tamnes, C. K., Walhovd, K. B., Grydeland, H., Holland, D., Østby, Y., Dale, A. M., et al. (2013). Longitudinal working memory development is related to structural 
maturation of frontal and parietal cortices. J. Cogn. Neurosci. 25, 1611-1623. doi: 10.1162/jocn_a_00434

Thiebaut de Schotten, M., Dell'Acqua, F., and Forkel, S. (2011a). A lateralized brain network for spatial attention. Nat. Neurosci. 14, 1245-1246.

Thiebaut de Schotten, M., Dell'Acqua, F., Valabregue, R., and Catani, M. (2012). Monkey to human comparative anatomy of the frontal lobe association tracts. Cortex 48, 82-96. doi: 10.1016/j.cortex.2011.10.001

Thiebaut de Schotten, M., Ffytche, D. H., Bizzi, A., Dell'Acqua, F., Allin, M., Walshe, M., et al. (2011b). Atlasing location, asymmetry and inter-subject variability of WM tracts in the human brain with MR diffusion tractography. Neuroimage 54, 49-59. doi: 10.1016/j.neuroimage.2010.07.055

Thomas, C., Avram, A., Pierpaoli, C., and Baker, C. (2015). Diffusion MRI properties of the human uncinate fasciculus correlate with the ability to learn visual associations. Cortex 72, 65-78. doi: 10.1016/j.cortex.2015.01.023

Thomas, C., Walker, L., Pierpaoli, C., and Baker, C. (2012). The role of the uncinate fasciculus in human visual-associative learning. J. Vis. 12, 1189-1189. doi: 10.1167/12.9.1189

Tozer, D., Ramani, A., Barker, G. J., Davies, G. R., Miller, D. H., and Tofts, P. S. (2003). Quantitative magnetization transfer mapping of bound protons in multiple sclerosis. Magn. Reson. Med. 50, 83-91. doi: 10.1002/mrm.10514

Treble, A., Hasan, K. M., Iftikhar, A., Stuebing, K. K., Kramer, L. A., Cox, C. S., et al. (2013). Working memory and corpus callosum microstructural integrity after pediatric traumatic brain injury: a diffusion tensor tractography study. J. Neurotrauma 30, 1609-1619. doi: 10.1089/neu.2013.2934

Tu, T.-W., Kim, J. H., Yin, F. Q., Jakeman, L. B., and Song, S.-K. (2013). The impact of myelination on axon sparing and locomotor function recovery in spinal cord injury assessed using diffusion tensor imaging. NMR Biomed. 26, 1484-1495. doi: $10.1002 / \mathrm{nbm} .2981$

Tuch, D. S., Salat, D. H., Wisco, J. J., Zaleta, A. K., Hevelone, N. D., and Rosas, H. D. (2005). Choice reaction time performance correlates with diffusion anisotropy in WM pathways supporting visuospatial attention. PNAS 102, 12212-12217. doi: $10.1073 /$ pnas. 0407259102

Turner, R. (2019). Myelin and modeling: bootstrapping cortical microcircuits. Front. Neural Circuits 13:34. doi: 10.3389/fncir.2019.00034

Urger, S. E., De Bellis, M. D., Hooper, S. R., Woolley, D. P., Chen, S. D., and Provenzale, J. (2015). The superior longitudinal fasciculus in typically developing children and adolescents: diffusion tensor imaging and neuropsychological correlates. J. Child Neurol. 30, 9-20. doi: 10.1177/ 0883073813520503

Valdés Hernández, M., del, C., Booth, T., Murray, C., Gow, A. J., Penke, L., et al. (2013). Brain WM damage in aging and cognitive ability in youth and older age. Neurobiol. Aging 34, 2740-2747. doi: 10.1016/j.neurobiolaging.2013.05.032

Valk, J., and Knaap, M. S. (2013). Magnetic Resonance of Myelin, Myelination, and Myelin Disorders. Berlin: Springer Science \& Business Media.

van der Holst, H. M., Tuladhar, A. M., van Norden, A. G. W., de Laat, K. F., van Uden, I. W. M., van Oudheusden, L. J. B., et al. (2013). Microstructural integrity of the cingulum is related to verbal memory performance in elderly with cerebral small vessel disease: the RUN DMC study. Neuroimage 65, 416-423. doi: 10.1016/j.neuroimage.2012.09.060

van Eimeren, L., Niogi, S., Mccandliss, B., Holloway, I., and Ansari, D. (2008). WM microstructures underlying mathematical abilities in children. Neuroreport 19, 1117-1121. doi: 10.1097/WNR.0b013e328307f5c1

Vandermosten, M., Boets, B., Poelmans, H., Sunaert, S., Wouters, J., and Ghesquière, P. (2012). A tractography study in dyslexia: neuroanatomic correlates of orthographic, phonological and speech processing. Brain 135, 935-948. doi: 10.1093/brain/awr363

Varma, G., Duhamel, G., de Bazelaire, C., and Alsop, D. C. (2015). Magnetization transfer from inhomogeneously broadened lines: a potential marker for myelin. Magn. Reson. Med. 73, 614-622. doi: 10.1002/mrm.25174

Varriano, F., Pascual-Diaz, S., and Prats-Galino, A. (2018). When the FAT goes wide: right extended Frontal Aslant Tract volume predicts performance on working memory tasks in healthy humans. PLoS One 13:e0200786. doi: 10.1371/ journal.pone. 0200786

Vassal, F., Boutet, C., Lemaire, J.-J., and Nuti, C. (2014). New insights into the functional significance of the frontal aslant tract: an anatomo-functional study using intraoperative electrical stimulations combined with diffusion tensor imaging-based fiber tracking. Br. J. Neurosurg. 28, 685-687. doi: 10.3109/ 02688697.2014.889810
Vedantam, A., Jirjis, M., Eckhardt, G., Sharma, A., Schmit, B. D., Wang, M. C., et al. (2013). Diffusion tensor imaging of the spinal cord: a review. Coluna/Columna 12, 64-69. doi: 10.1590/\$1808-18512013000100014

Vogt, B. A., Vogt, L., Farber, N. B., and Bush, G. (2005). Architecture and neurocytology of monkey cingulate gyrus. J. Comp. Neurol. 485, 218-239. doi: $10.1002 /$ cne.20512

Vogt, C., and Vogt, O. (1919). Allgemeinere ergebnisse unserer hirnforschung. J. Psychol. Neurol. 25, 279-468.

Von Der Heide, R. J., Skipper, L. M., Klobusicky, E., and Olson, I. R. (2013). Dissecting the uncinate fasciculus: disorders, controversies and a hypothesis. Brain 136, 1692-1707. doi: 10.1093/brain/awt094

Walsh, M., Montojo, C. A., Sheu, Y.-S., Marchette, S. A., Harrison, D. M., Newsome, S. D., et al. (2011). Object working memory performance depends on microstructure of the frontal-occipital fasciculus. Brain Connect 1, 317-329. doi: 10.1089/brain.2011.0037

Wang, Y., Fernández-Miranda, J. C., Verstynen, T., Pathak, S., Schneider, W., and Yeh, F.-C. (2013). Rethinking the role of the middle longitudinal fascicle in language and auditory pathways. Cereb. Cortex 23, 2347-2356. doi: 10.1093/ cercor/bhs 225

Wang, Y., Wang, Q., Haldar, J. P., Yeh, F.-C., Xie, M., Sun, P., et al. (2011). Quantification of increased cellularity during inflammatory demyelination. Brain 134, 3590-3601. doi: 10.1093/brain/awr307

Waxman, S. G. (1975). Electron-microscopic observations on preterminal fibers in the oculomotor nucleus of the cat: with special reference to the relation between axon diameter and myelin thickness in mammalian gray matter. J. Neurol. Sci. 26, 395-400. doi: 10.1016/0022-510X(75)90210-5

Waxman, S. G. (1980). Determinants of conduction velocity in myelinated nerve fibers. Muscle Nerve. 3, 141-150. doi: 10.1002/mus.880030207

Webb, W. G. (2017). "Central language mechanism and learning," in Neurology for the Speech-Language Pathologist, Sixth Edn, Chap. 9, ed. W. G. Webb (Maryland heights, MO: Mosby), 181-205. doi: 10.1016/B978-0-323-10027-4.00009-9

Westerhausen, R., Walter, C., Kreuder, F., Wittling, R. A., Schweiger, E., and Wittling, W. (2003). The influence of handedness and gender on the microstructure of the human CC: a diffusion-tensor magnetic resonance imaging study. Neurosci. Lett. 351, 99-102. doi: 10.1016/j.neulet.2003.07.011

Westin, C.-F., Maier, S. E., Mamata, H., Nabavi, A., Jolesz, F. A., and Kikinis, R. (2002). Processing and visualization for diffusion tensor MRI. Med. Image Anal. 6, 93-108. doi: 10.1016/s1361-8415(02)00053-1

Westlye, L. T., Walhovd, K. B., Dale, A. M., Bjørnerud, A., Due-Tønnessen, P., Engvig, A., et al. (2010). Life-span changes of the human brain WM: diffusion tensor imaging (DTI) and volumetry. Cereb. Cortex 20, 2055-2068. doi: 10. 1093/cercor/bhp280

White, N. S., Leergaard, T. B., D’Arceuil, H., Bjaalie, J. G., and Dale, A. M. (2013). Probing tissue microstructure with restriction spectrum imaging: Histological and theoretical validation. Hum. Brain Mapp. 34, 327-346. doi: 10.1002/hbm. 21454

Whittall, K. P., MacKay, A. L., Graeb, D. A., Nugent, R. A., Li, D. K., and Paty, D. W. (1997). In vivo measurement of T2 distributions and water contents in normal human brain. Magn. Reson. Med. 37, 34-43. doi: $10.1002 / \mathrm{mrm} .1910370107$

Williamson, J. M., and Lyons, D. A. (2018). Myelin dynamics throughout life: an ever-changing landscape? Front. Cell Neurosci. 12:424. doi: 10.3389/fncel.2018. 00424

Wu, T. C., Wilde, E. A., Bigler, E. D., Yallampalli, R., McCauley, S. R., Troyanskaya, M., et al. (2010). Evaluating the relationship between memory functioning and cingulum bundles in acute mild traumatic brain injury using diffusion tensor imaging. J. Neurotrauma 27, 303-307. doi: 10.1089/neu.2009. 1110

Wu, Y., Sun, D., Wang, Y., and Wang, Y. (2016). Subcomponents and connectivity of the inferior fronto-occipital fasciculus revealed by diffusion spectrum imaging fiber tracking. Front. Neuroanat. 10:88. doi: 10.3389/fnana.2016. 00088

Xin, J., Zhang, Y., Tang, Y., and Yang, Y. (2019). Brain differences between men and women: evidence from deep learning. Front. Neurosci. 13:185. doi: 10.3389/ fnins. 2019.00185

Xydis, V., Astrakas, L., Zikou, A., Pantou, K., Andronikou, S., and Argyropoulou, M. I. (2006). Magnetization transfer ratio in the brain of preterm subjects: 
age-related changes during the first 2 years of life. Eur. Radiol. 16, 215-220. doi: 10.1007/s00330-005-2796-8

Yakovlev, P., and Lecours, A. (1967). "The myelogenetic cycles of regional maturation of the brain," in Regional Development of the Brain in early Life, ed. A. Minkowski (Philadelphia, PA: Davis Co), 3-70.

Yakovlev, P. I., and Locke, S. (1961). Limbic nuclei of thalamus and connections of limbic cortex. III. Corticocortical connections of the anterior cingulate gyrus, the cingulum, and the subcallosal bundle in monkey. Arch. Neurol. 5, 364-400. doi: 10.1001/archneur.1961.00450160014002

Yarnykh, V. L. (2002). Pulsed Z-spectroscopic imaging of crossrelaxation parameters in tissues for human MRI: theory and clinical applications. Magn. Reson. Med. 47, 929-939. doi: 10.1002/mrm. 10120

Yarnykh, V. L. (2012). Fast macromolecular proton fraction mapping from a single off-resonance magnetization transfer measurement. Magn. Reson. Med. 68, 166-178. doi: 10.1002/mrm.23224

Yarnykh, V. L., Prihod'ko, I. Y., Savelov, A. A., and Korostyshevskaya, A. M. (2018). Quantitative assessment of normal fetal brain myelination using fast macromolecular proton fraction mapping. AJNR Am. J. Neuroradiol. 39, 13411348. doi: 10.3174/ajnr.A5668

Yarnykh, V. L., and Yuan, C. (2004). Cross-relaxation imaging reveals detailed anatomy of white matter fiber tracts in the human brain. Neuroimage 23, 409-424. doi: 10.1016/j.neuroimage.2004.04.029

Yeatman, J. D., Wandell, B. A., and Mezer, A. A. (2014). Lifespan maturation and degeneration of human brain white matter. Nat. Commun. 5:4932. doi: 10.1038/ncomms5932

Yu, J., Lam, C. L. M., and Lee, T. M. C. (2017). WM microstructural abnormalities in amnestic mild cognitive impairment: a meta-analysis of whole-brain and
ROI-based studies. Neurosci. Biobehav. Rev. 83, 405-416. doi: 10.1016/j. neubiorev.2017.10.026

Zemmoura, I., Herbet, G., Moritz-Gasser, S., and Duffau, H. (2015). New insights into the neural network mediating reading processes provided by corticosubcortical electrical mapping. Hum. Brain Mapp. 36, 2215-2230. doi: 10.1002/ hbm. 22766

Zhang, H., Schneider, T., Wheeler-Kingshott, C. A., and Alexander, D. C. (2012). NODDI: Practical in vivo neurite orientation dispersion and density imaging of the human brain. NeuroImage 61, 1000-1016. doi: 10.1016/j.neuroimage.2012. 03.072

Zhang, S., Wang, Y., Deng, F., Zhong, S., Chen, L., Luo, X., et al. (2018). Disruption of superficial WM in the emotion regulation network in bipolar disorder. Neuroimage Clin. 20, 875-882. doi: 10.1016/j.nicl.2018.09.024

Zhang, Y., Zhang, J., Oishi, K., Faria, A. V., Jiang, H., Li, X., et al. (2010). Atlasguided tract reconstruction for automated and comprehensive examination of the WM anatomy. Neuroimage 52, 1289-1301. doi: 10.1016/j.neuroimage.2010. 05.049

Conflict of Interest: The authors declare that the research was conducted in the absence of any commercial or financial relationships that could be construed as a potential conflict of interest.

Copyright $(02021$ Buyanova and Arsalidou. This is an open-access article distributed under the terms of the Creative Commons Attribution License (CC BY). The use, distribution or reproduction in other forums is permitted, provided the original author(s) and the copyright owner(s) are credited and that the original publication in this journal is cited, in accordance with accepted academic practice. No use, distribution or reproduction is permitted which does not comply with these terms. 